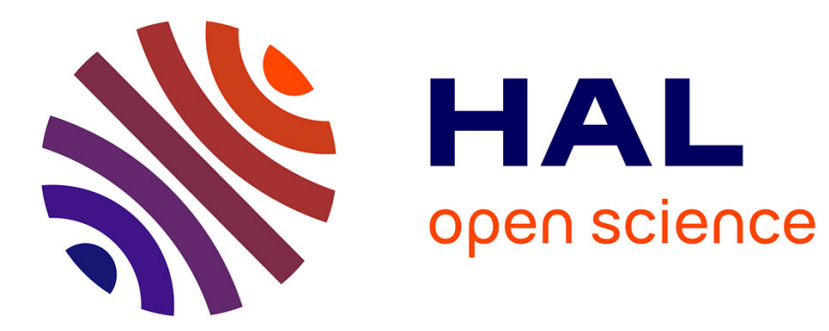

\title{
Le Bois Pargas, à Pageas (Haute-Vienne) : un nouveau témoin du Néolithique final en Limousin.
}

Thomas Perrin, Ewen Ihuel, Hugues Plisson

\section{To cite this version:}

Thomas Perrin, Ewen Ihuel, Hugues Plisson. Le Bois Pargas, à Pageas (Haute-Vienne) : un nouveau témoin du Néolithique final en Limousin.. Bulletin de la Société préhistorique française, 2007, 104 (3), pp.543-563. 10.3406/bspf.2007.13591 . hal-00266618

\section{HAL Id: hal-00266618 https://hal.science/hal-00266618}

Submitted on 14 Oct 2012

HAL is a multi-disciplinary open access archive for the deposit and dissemination of scientific research documents, whether they are published or not. The documents may come from teaching and research institutions in France or abroad, or from public or private research centers.
L'archive ouverte pluridisciplinaire HAL, est destinée au dépôt et à la diffusion de documents scientifiques de niveau recherche, publiés ou non, émanant des établissements d'enseignement et de recherche français ou étrangers, des laboratoires publics ou privés. 


\title{
Le Bois Pargas à Pageas (Haute-Vienne): un nouveau témoin \\ Thomas PERRIN, Ewen IHUEL et Hugues PLISSON du Néolithique final en Limousin
}

\begin{abstract}
Résumé
Le gisement du Bois Pargas, situé sur la commune de Pageas en HauteVienne, fut identifié à la suite des violentes tempêtes de la fin de l'année 1999 par la découverte de matériel archéologique pris dans une souche de châtaigner renversée. La réalisation d'un sondage permit de montrer que cet arbre s'était enraciné sur une fosse préhistorique. Bien qu'aucun vestige organique n'ait été conservé, le type de matériel archéologique découvert permet d'interpréter cette structure comme le témoin d'un monument funéraire pseudo-mégalithique. La fosse sépulcrale contenait en effet un ensemble de six poignards en silex du Grand-Pressigny, associés à des pointes de flèches, des scies à coches, de la céramique et des éléments de parure. Ce matériel permet de situer le gisement au III millénaire avant notre ère et l'analyse technologique des poignards met en évidence deux époques de production qui conduisent à s'interroger sur la durée de fonctionnement de la structure funéraire.
\end{abstract}

\begin{abstract}
The archaeological site of «Bois Pargas», near the village of Pageas in Haute-Vienne (Limousin, France), was discovered after the violent storms of the end of 1999, by the finding of artefacts in the roots of a fallen chestnut tree. A small excavation revealed that the tree had grown on a Neolithic pit. Although no organic remains have been preserved, the archaeological artefacts allowed the structure to be interpreted as a pseudo-megalithic funerary monument. The burial pit contained six Grand Pressigny flint daggers, together with arrowheads, "notched saws», ceramics and some ornaments. All these artefacts are typical of the 3rd millennium BC and technological analysis of the daggers points to two production periods which leads to discussion regarding the duration of use of the funerary structure.
\end{abstract}

\section{INTRODUCTION}

À la fin de l'année 1999, une violente tempête a largement ravagé une grande partie septentrionale du territoire français. Durant son périple, elle a heurté de plein fouet les forêts limousines, créant de vastes et impressionnants dégâts. Nombre de gisements archéologiques ont alors été non seulement enfouis sous un enchevêtrement d'arbres brisés, mais aussi en partie détruits, ou en tous cas sérieusement abîmés par l'arrachement complet de certains de ces arbres. C'est lors 
de l'examen d'une souche de châtaigner renversée, au printemps suivant, que M. Jacques Devalette, membre de l'association ArchéA ${ }^{1}$ de Limoges, découvrit le gisement du Bois Pargas par le ramassage de quelques objets archéologiques remarquables. Quelques mois plus tard, nous avons procédé à une reconnaissance du site, en compagnie de son inventeur et de quelques membres de l'association ArchéA. Le gisement, à ce moment-là, se caractérisait principalement par un tas d'épierrement important, situé en bordure d'un vaste plateau, et au centre duquel émergeait, subverticale, une imposante dalle de gneiss. Un nouvel examen de

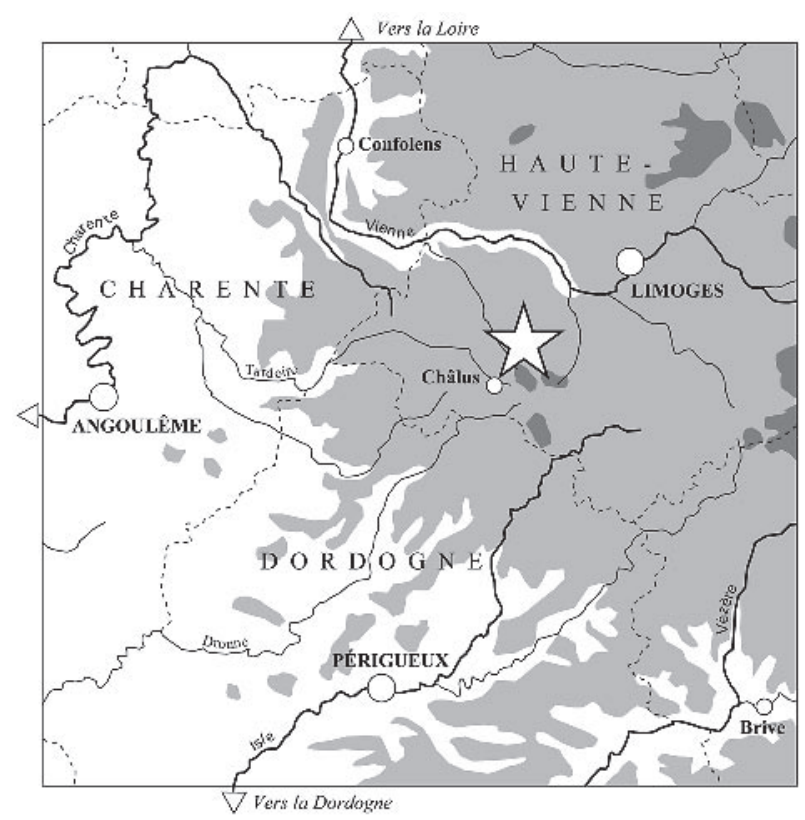

Fig. 1 - Localisation du gisement du Bois Pargas, Pageas, HauteVienne. la souche renversée entraîna la découverte d'objets préhistoriques, notamment quelques fragments de poignards en silex du Grand-Pressigny. Ces simples ramassages, ponctuels, permirent de constituer une série déjà exceptionnelle et pouvaient laisser penser, au vu de l'emplacement et de l'aspect du gisement, que nous étions là en présence d'une structure funéraire mégalithique de la fin du Néolithique. Il nous a alors paru opportun, d'une part de vérifier cette hypothèse, et d'autre part de caractériser le contexte de découverte de ces poignards par la réalisation d'un sondage. Celuici a été rendu possible grâce à la collaboration efficace du service régional de l'Archéologie de Limoges et grâce à l'investissement humain et matériel de l'association ArchéA $^{2}$. Ce sondage s'inscrivait dans la continuité d'opérations préalables, notamment une première prospection-inventaire menée sur l'ensemble des monts de Châlus (Conte et Perrin, 1998a et b), elle-même poursuivie par une prospection thématique plus spécifiquement consacrée aux occupations préhistoriques de cette région (Perrin, 2000).

\section{PRÉSENTATION GÉNÉRALE DES VESTIGES}

Le gisement se situe sur la commune de Pageas (Haute-Vienne), à une vingtaine de kilomètres au sudouest de Limoges (coordonnées Lambert zone II étendue $: x=499.150, y=2076.650, z=414$ NGF, fig. 1), à proximité du hameau des Fosses, au lieu-dit cadastral Bois Pargas. Il est installé sur la bordure orientale d'un vaste plateau métamorphique dont le substrat est composé de gneiss schisteux à biotite, parfois affleurant et, comme l'a révélé notre sondage, de filons de leptynites massives à grains moyens et biotite, inclus dans le socle gneissique. La végétation actuelle du plateau est composée en parts à peu près égales de prés, où

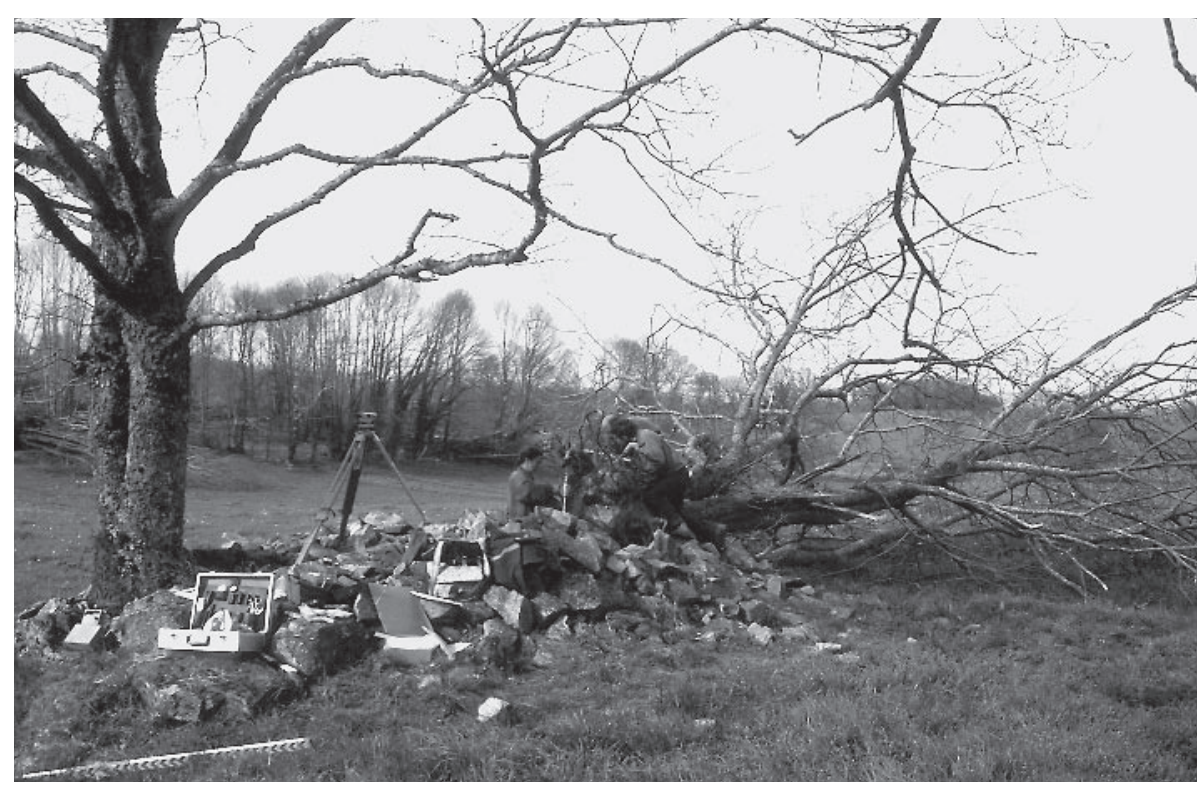

Fig. 2 - Aperçu du site au printemps 2000, peu de temps après sa découverte. On aperçoit au premier plan le tas d'épierrement moderne ainsi que le châtaigner, arraché par la tempête, dans la souche duquel ont été découverts plusieurs objets archéologiques, et notamment des fragments de poignards en silex du GrandPressigny. 
paissent quelques belles limousines, et de bois dans lesquels l'essence forestière principalement représentée est le châtaigner.

La souche était implantée au sein d'un tertre de pierres irrégulier, suggérant de prime abord la présence en ce lieu d'un tumulus. En fait, nous avons rapidement pu montrer qu'il ne s'agissait là que d'un tas d'épierrement moderne (US 1003, fig. 2). Cette datation est assurée non seulement par l'aspect même de ce tertre (irrégulier, non délimité...), mais aussi par l'absence de sédiment interstitiel entre les pierres qui le constituent ainsi que par le témoignage même du propriétaire du terrain ${ }^{3}$. La localisation de ce tas d'épierrement, dans la zone centrale du pré, s'explique en réalité par une contrainte géologique, qui est celle de la présence sous-jacente, en cet endroit précis, d'un affleurement rocheux. Celui-ci définit une butte ovalaire d'environ $20 \mathrm{~m}$ de long pour une douzaine de large, et de près d'un mètre de puissance par rapport au niveau général du pré. La présence de cette roche affleurante a interdit tout travail agricole en profondeur dans cette partie de la parcelle qui fut alors mise à profit pour le stockage des pierres arrachées ailleurs. Cette gestion opportuniste d'une contrainte géologique a probablement
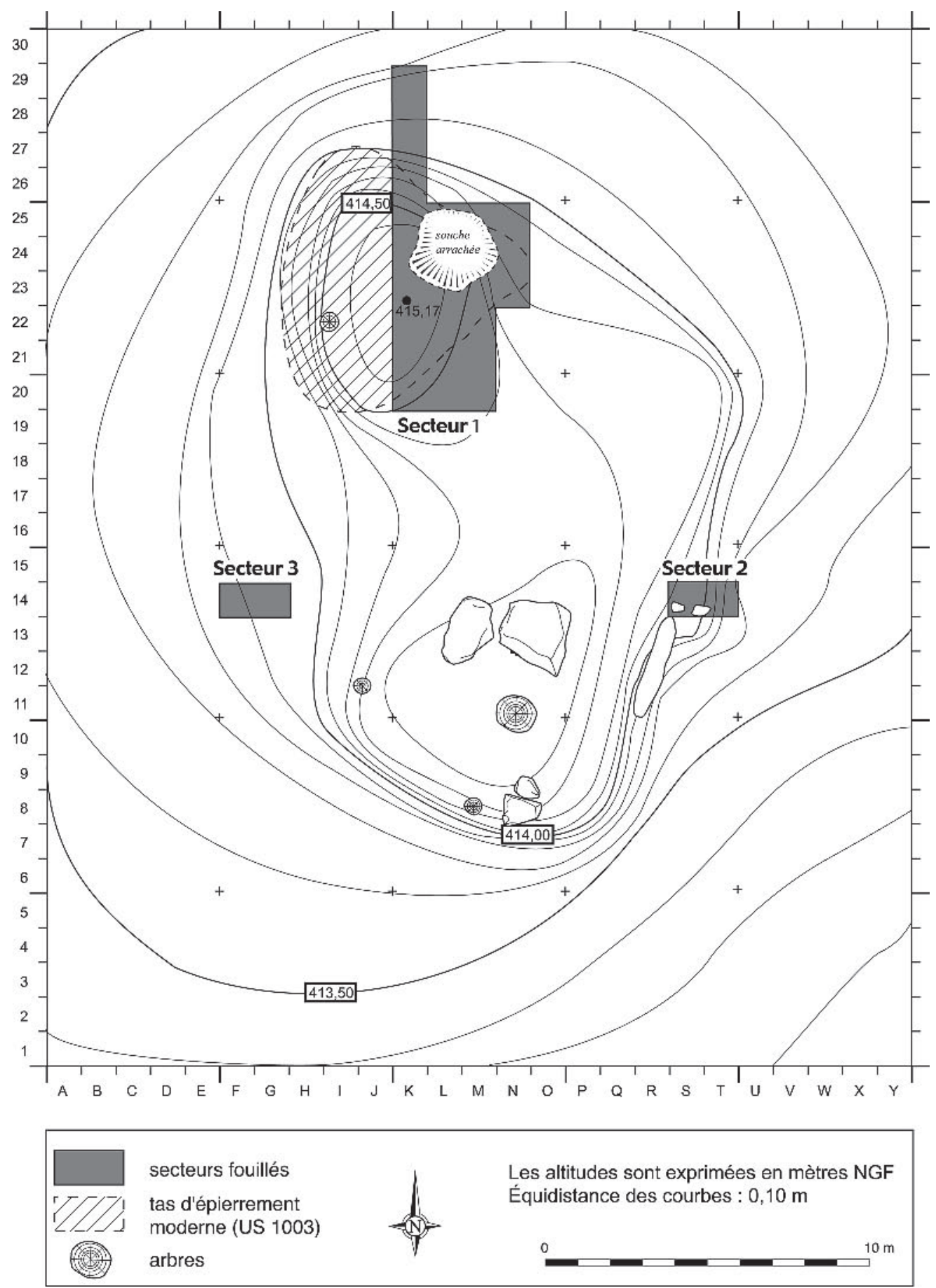

Fig. 3 - Plan de masse du site et localisation des trois secteurs sondés. Les courbes de niveau illustrent le fait que le site est placé dans la partie sommitale d'une légère éminence naturelle. 
assuré une certaine protection au gisement archéologique.

Le sondage a montré qu'aucune structure préhistorique construite en élévation n'était conservée. Seuls sont attestés des aménagements en creux. Ceux-ci

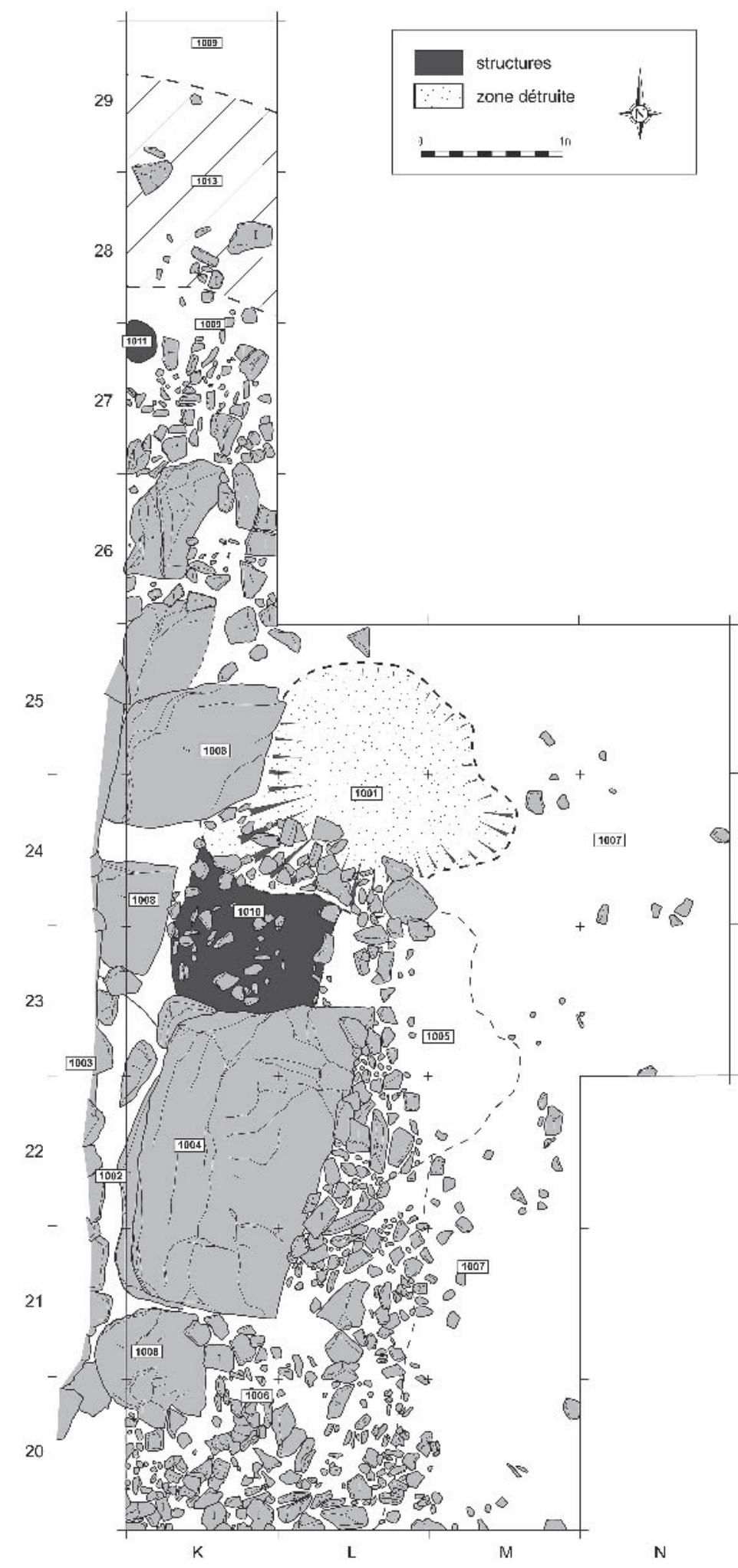

Fig. 4 - Relevé général du secteur 1. La principale structure est composée par l'US 1010 dont la partie septentrionale a été détruite par l'arrachement de l'arbre (US 1001). Cette fosse vient s'appuyer au sud contre le bloc de gneiss 1004 . restent cependant difficilement perceptibles de par le faible remplissage général, mais aussi à cause des perturbations importantes dues à la présence de cinq arbres. Le châtaigner effondré était situé à l'endroit même de la principale structure archéologique et son arrachement accidentel en a emporté près de la moitié. L'attention a bien sûr essentiellement porté sur les environs immédiats de la souche et le tas d'épierrement (fig. 3). Ce secteur, baptisé secteur 1, comprend une surface de $25 \mathrm{~m}^{2}$ (mètres carrés K-L/20-29) et fut le plus riche d'enseignements. Le secteur 2 a été implanté en R-T/14, sur une surface de $2,5 \mathrm{~m}^{2}$. Sa localisation a été déterminée par la présence d'une nette rupture de pente, dans le but d'en définir l'origine anthropique ou non. Sur la même bande 14, dans les mètres carrés $\mathrm{F}$ et $\mathrm{G}$, le secteur 3 répondait au même souci, mais pour le versant ouest de la butte. Il n'a concerné que $2 \mathrm{~m}^{2}$. Dans chacun des trois secteurs ouverts, la fouille a été menée jusqu'au substrat et seuls les secteurs 1 et 2 ont livré des vestiges archéologiques.

\section{LA FOUILLE : PRÉSENTATION DES DONNÉES DE TERRAIN}

\section{Le secteur 1}

Ce secteur est celui sur lequel a porté la plus grande part de nos efforts (fig. 4). La situation topographique particulière (élévation géologique sur un rebord de plateau) a entraîné une puissance sédimentaire relativement restreinte, de l'ordre de $0,30 \mathrm{~m}$ de moyenne. Les perturbations végétales tendent de plus à gommer la lisibilité des dépôts et des structures rencontrés. L'interprétation du gisement repose donc sur des données partielles et relativement fugaces.

D'un point de vue stratigraphique, le remplissage est peu dilaté et présente une succession de couches globalement planes. Dans la moitié sud du secteur 1 (K-M/20-22), on trouve, sous l'humus 1002, les deux US 1006 et 1007 . Si cette dernière ne présentait aucune trace anthropique, l'US 1006 (de même que son extension septentrionale 1005) contenait du mobilier ainsi que de rares microcharbons épars. Proches d'un point de vue sédimentaire, il semble en fait que ces trois US 1005, 1006 et 1007 ne soient que des variations latérales d'une même couche limoneuse, plus anthropisée dans sa partie occidentale (US 1005 et 1006).

Dans l'ensemble de ce premier secteur, seules deux structures ont pu être identifiées. La première correspond à un trou de poteau, dénommé PO 001. Situé à la limite $\mathrm{K} / 27-28$, il a un diamètre de $20 \mathrm{~cm}$ environ, une forme tronconique et une profondeur de $20 \mathrm{~cm}$. Son remplissage (US 1011) est un limon meuble et homogène dont la couleur brun-noir, légèrement foncée, traduit la présence à l'origine de composants organiques et tranche nettement avec l'encaissant sableux 1009. Cette structure, localisée et relativement isolée, n'a pas livré de mobilier archéologique.

Plus au nord, en K/28-29, l'US 1013 correspond à une petite variation de couleur de l'US 1009. Large d'environ $1 \mathrm{~m}$ et de délinéation semble-t-il courbe, 
nous avions émis sur le terrain l'hypothèse qu'il puisse s'agir d'un fossé périphérique. La fouille n'a cependant pu montrer l'existence d'un réel creusement à cet endroit, malgré la persistance sur plusieurs centimètres d'une coloration distincte. Localisée dans la rupture de pente et à la limite de la zone d'exploitation agricole, nous ne savons donc pas s'il s'agit là d'un fait archéologique véritable ou d'une variation latérale de faciès de l'US 1009, hypothèse cependant la plus probable.

La fosse FS 003 constitue le principal fait archéologique du secteur 1, comme du site en général. La souche arrachée 1001 en a détruit la partie nord, l'amputant d'une bonne moitié environ. Ses racines en ont de plus perturbé partiellement les contours. On peut cependant reconstituer une fosse subrectangulaire d'axe nord-sud, de près de $0,80 \mathrm{~m}$ de large pour environ $1 \mathrm{~m}$ de longueur conservée (de probablement au moins $2 \mathrm{~m}$ de long à l'origine). Sa profondeur observée est de 0,30 m. Cette fosse a été creusée immédiatement au nord de la grande dalle de gneiss affleurante 1004. Son fond a été aménagé par l'insertion transversale d'un gros bloc de gneiss, disposé plus ou moins horizontalement entre deux dalles obliques, inclinées selon le pendage naturel, conférant ainsi un aspect «en baignoire» à cette structure (fig. 5). L'essentiel du

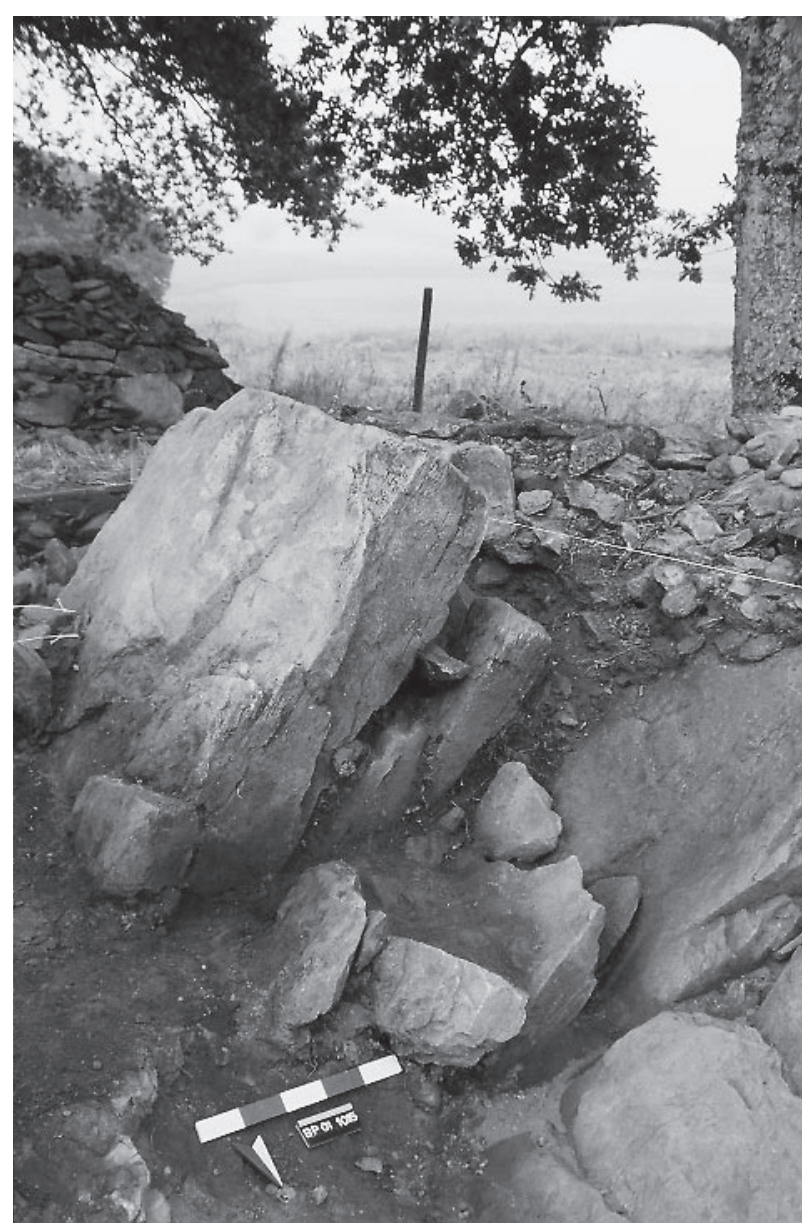

Fig. 5 - Vue de la structure excavée 1010 en fin de fouille et du bloc de gneiss 1004. Au premier plan, on aperçoit la base de la partie détruite lors de l'arrachement du châtaigner durant la tempête. mobilier archéologique découvert sur le gisement provient de la partie inférieure de cette fosse. Le tout fut colmaté par un limon brun-noir organique (US 1010).

S'il n'existe pas d'évidence de structure de couverture conservée, il faut cependant remarquer que la grande dalle de gneiss 1004 était déjà affleurante à l'époque préhistorique. Sa position est parfaitement naturelle, comme le démontrent son pendage, identique au pendage général du substrat gneissique de l'ensemble du plateau, ainsi que la présence de diaclases et d'axes de délitement eux aussi similaires. D'autres affleurements locaux du substrat sont attestés en plusieurs endroits du plateau, reproduisant des buttes rocheuses similaires, quoique moins marquées. La plupart s'organise également selon un axe grossièrement nord-sud, à l'instar de celui identifié dans le secteur 1 . Ce dernier se distingue cependant par l'aspect relativement parallélépipédique de la dalle principale 1004, alors qu'elle aurait naturellement dû se prolonger plus avant vers le nord, suivant le banc 1008 dont elle est l'un des constituants. On peut donc émettre l'hypothèse que l'extrémité septentrionale de cet affleurement en général (US 1008) et de la dalle 1004 en particulier a été volontairement aménagée, réduite, afin de pouvoir accueillir la structure excavée. Les très nombreux fragments de gneiss présents dans les unités stratigraphiques 1005 et 1006 (qui, rappelons-le, livrent également du mobilier préhistorique) pourraient être compris comme des débris produits lors de cet aménagement. Cependant, l'absence de toutes traces nettes sur la dalle ou celle de stigmates de percussion sur les fragments de gneiss eux-mêmes ne permettent pas de confirmer de manière absolue cette hypothèse. Mais, dans tous les cas, cette anomalie géologique locale remarquable a été mise à profit, plus ou moins façonnée, avant de recevoir un aménagement construit.

Le secteur 1 témoigne donc de la présence, sur ce bord de plateau, d'une fosse rectangulaire de bonne taille. La présence concomitante d'une dalle de gneiss affleurante au sommet d'une butte naturelle lui confère un aspect «pseudo-mégalithique» et constitue de fait un marquage aérien nettement visible dans le paysage. De plus, il est possible que cette dalle ait été aménagée volontairement. La présence proche d'un trou de poteau et de tessons épars (US 1006) témoigne de l'existence d'aménagements connexes, conférant au gisement un caractère plus complexe que celui d'une simple fosse isolée.

\section{Le secteur 2}

Implanté en R-T/14 (fig. 6), la fouille de ce secteur avait pour but d'identifier la nature du décrochement général constaté en périphérie du secteur 1. Elle a mis en évidence la présence d'un niveau archéologique complexe, notamment en R/14. Celui-ci correspond aux US 2005, 2008-2010 (PO 002) et 2006. Le niveau 2007 est un niveau géologique au sein duquel du matériel archéologique a percolé depuis les niveaux susjacents. 

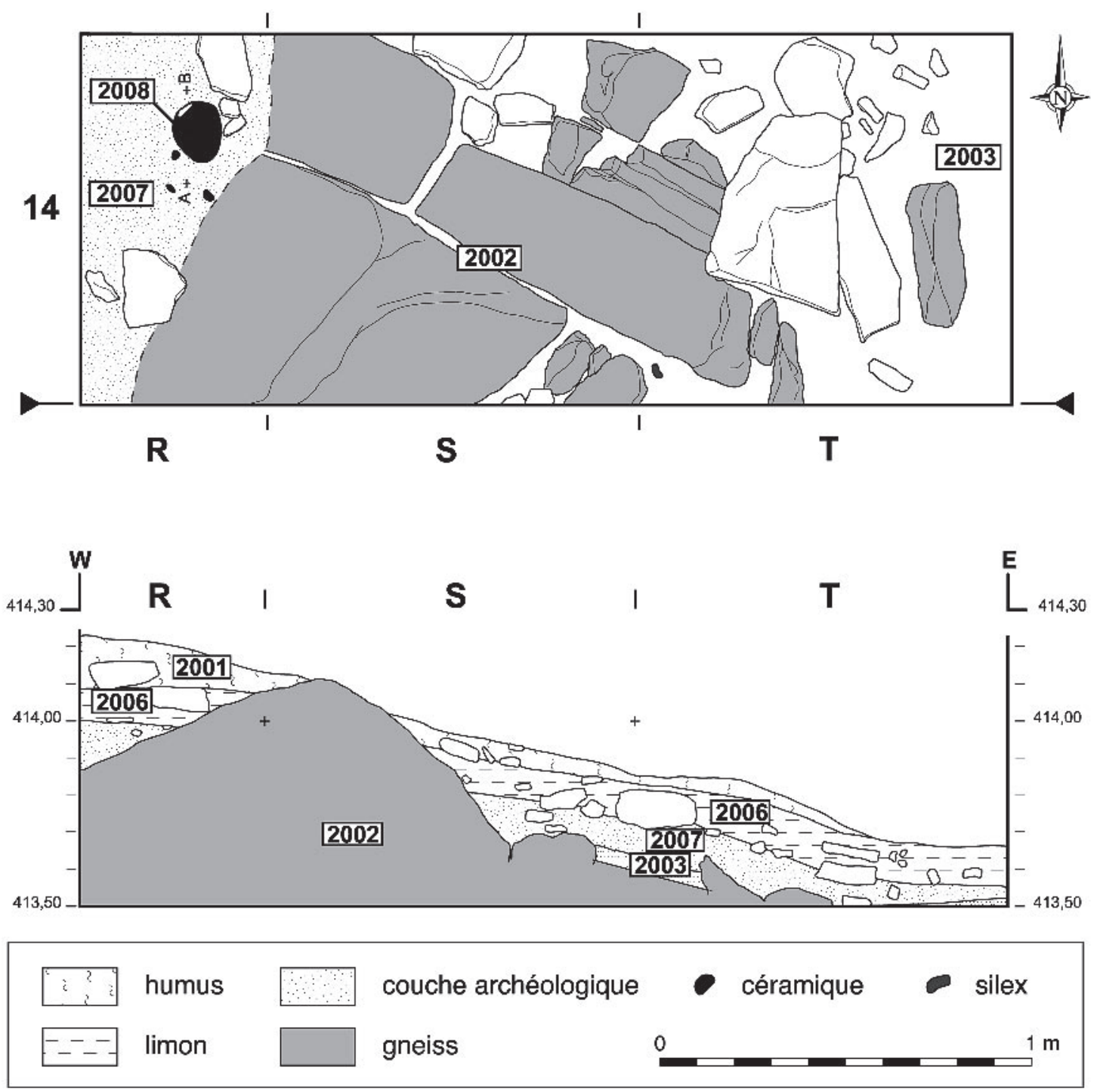

Fig. 6 - Plan et coupe stratigraphique (ligne 13-14) du secteur 2. L'US 2008 correspond à une structure excavée (trou de poteau PO 002).

Le niveau archéologique se caractérise par la présence du trou de poteau PO 002, creusé aux dépens des US 2006 et 2007. Situé au bord du décrochement du substrat, il est de forme globalement tronconique et mesure environ $20 \mathrm{~cm}$ de diamètre pour une profondeur conservée équivalente. Après sa désaffection, il a été recouvert par l'US 2005, correspondant à un niveau de dallettes gneissiques subhorizontales. Dégagé sur seulement $0,5 \mathrm{~m}^{2}$, il est difficile de savoir s'il s'agit d'un aménagement volontaire ou non de cette partie de l'éminence. Il peut s'agir là soit d'un effet aléatoire local, naturel, soit d'un témoignage discret d'un aménagement anthropique en liaison avec le trou de poteau PO 002.

Hormis un silex patiné en T/14, d'une matière inédite ailleurs dans le gisement, aucun indice d'occupation préhistorique n'a été découvert dans et au-delà de la rupture de pente.
En définitive, le secteur 2 atteste de l'extension des aménagements préhistoriques à l'ensemble de l'éminence. La présence d'un trou de poteau incontestable, de microcharbons, de fragments de céramiques et d'un niveau de dallettes peut même suggérer la présence d'une structure construite au sens le plus large du terme : du simple aménagement localisé à la réelle structure construite.

\section{Le secteur 3}

Fouillé dans la même optique que le secteur 2, le secteur $3 \mathrm{~s}$ 'avère plus pauvre en informations et aucun niveau archéologique net n'a pu y être déterminé. Seules les quelques pierres de l'US 3005 ressortent dans l'environnement sédimentaire de cet endroit du site, mais ne traduisent aucun aménagement net ou action anthropique quelconque. 


\section{LE MOBILIER ARCHÉOLOGIQUE}

\section{La céramique}

Le matériau céramique n'est représenté que par 27 tessons, totalisant 167 grammes (soit $6 \mathrm{~g}$ de moyenne), qui se répartissent sur l'ensemble du secteur 1 et du secteur 2 . Il s'agit généralement de petits tessons, voire de microtessons. La plupart provient de céramiques épaisses (environ $9 \mathrm{~mm}$ ) dont les surfaces ne sont pas conservées. Les pâtes sont de couleur brun-rouge et comportent un dégraissant minéral grossier (dont du quartz). Des traces de colombins sont parfois visibles sur les faces internes. D'un point de vue typologique, aucune forme complète n'est restituable. Seuls deux fragments de fond de vase aplanis permettent d'approcher la typologie des récipients.

Cinq autres fragments, répartis sur l'ensemble du site, montrent des parois plus fines, un dégraissant difficilement visible à l'œil nu et des surfaces extérieures probablement lissées. Ils témoignent de la présence d'une catégorie de céramique plus fine que la précédente, sans qu'il soit malheureusement possible de la documenter plus en détail.

Malgré l'absence de formes complètes, les éléments de fond ainsi que les caractères des pâtes rappellent indubitablement le Néolithique final de tradition artenacienne, bien attesté régionalement (Conte et Perrin, 1998a et b; Conte et al., 2001; Perrin, 2000).

\begin{tabular}{ll}
\hline & action longitudinale \\
& action transversale \\
& $\begin{array}{l}\text { traces techniques } \\
\text { (aménagement) } \\
\text { limite brutale d'usure } \\
\text { usure totalement recoupée } \\
\text { par la retouche } \\
\text { usure partiellement recoupée } \\
\text { par la retouche }\end{array}$
\end{tabular}

Matières travaillées:

10 - minérale

20 - végétale

21 - graminées

23 - non ligneux particulier

30 - animale tendre

31 - viande

32 - peau

50 - carcasse

Fig. 7 - Codes graphiques utilisés sur les figures 8 à 14 décrivant les traces d'utilisation observées.

\section{L'industrie lithique : aspects technotypologiques et tracéologiques}

Parmi les vingt-trois éléments lithiques découverts tant lors des phases de prospection que lors de la fouille, trois sont des éclats provenant de lames de haches polies en silex. Les vingt autres pièces appartiennent à l'industrie lithique taillée. Seul un petit fragment proximal d'éclat laminaire en silex blond translucide patiné provient du secteur 2. C'est l'unique élément de cette matière attesté sur le site. Au sein du secteur 1, l'US 1006 se singularise également par la présence isolée d'un petit nucléus irrégulier en jaspe. Là aussi, cette matière n'est représentée que par un élément.

Le reste de l'industrie provient de la fosse FS 003 (US 1001, 1010 et 1015, fig. 7 à 14). Les éléments les plus remarquables sont constitués par trois pointes de flèches à pédoncule et ailerons, ainsi que par six poignards et fragments. La plupart sont en silex du GrandPressigny, origine confirmée lors de l'examen de ces pièces par Nicole Mallet ${ }^{4}$. Parmi eux, trois paraissent se rapporter au faciès brun sombre de Larcy dans la moyenne vallée du Brignon (Aubry, 1991). Les autres éléments témoignent d'une certaine diversité, tout en restant dans des gammes brun clair assez communes dans la région (ibid.). Seul un fragment de lame pisciforme pourrait provenir des niveaux jurassiques d'Aquitaine (examen Pierrick Fouéré, fig. 12, nº 5).

- Poignard 1 (BP00-1001-10 et BP00-1001-11, fig. 8)

Le silex est de couleur cire, zoné de plages plus grises ou légèrement rouges. La pièce mesure $220 \mathrm{~mm}$ de long pour 40,5 $\mathrm{mm}$ de large et $10 \mathrm{~mm}$ d'épaisseur maximale. La flèche est peu importante; une ondulation assez marquée s'observe en partie distale. La face supérieure présente trois enlèvements laminaires dont le dernier, en position centrale, provient d'un plan de frappe opposé. Ces observations sont de bons arguments pour attribuer ce support à un nucléus à crêtes antérolatérales (ou production de type «NACAL» : Pelegrin et Ihuel, 2005, p. 46).

Un polissage important a été réalisé sur la moitié distale de la face supérieure, transversalement à l'axe de la pièce et selon une incidence légèrement oblique. Il ne subsiste que sous la forme de quatre petites plages polies en partie centrale. Diverses opérations sont visibles, notamment une retouche couvrante, étroite et assez irrégulière, sur la moitié distale de la lame, réalisée dans un sens senestre initié sur le bord gauche de la pointe et terminé sur le droit. Il s'agit vraisemblablement d'une reprise de l'objet, car la retouche du côté droit de l'extrémité apicale a emporté la plus grande part d'une usure de coupe végétale (fig. 14, photo 1). Parallèlement ou antérieurement, un aménagement bifacial de la moitié proximale a été réalisé par percussion tendre. La dernière opération est une régularisation des tranchants par des petits enlèvements courts réalisés par pression. Cette retouche est discontinue et n'affecte que quelques parties des bords. 

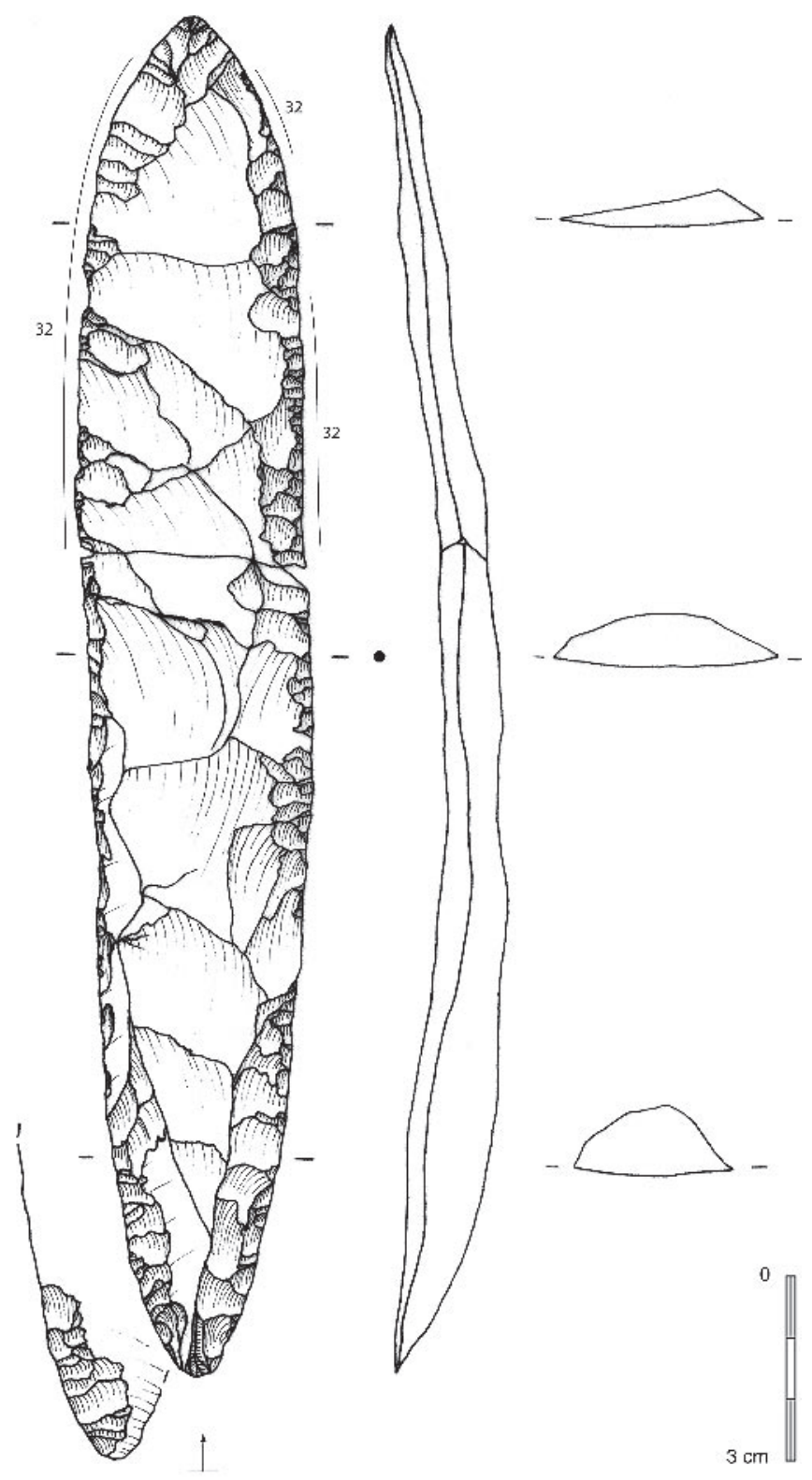

Fig. 8 - Poignard n 1 (BP00-1001-10 et BP00-1001-11). Poignard en silex du Grand-Pressigny, de couleur cire, témoignant d'une préparation de la face supérieure par polissage avant la retouche. Sa partie active, brisée, témoigne d'une utilisation pour trancher de la matière carnée ou cutanée tendre, après un stade antérieur de coupe végétale recoupé par la retouche apicale.

Ce poignard est brisé au premier tiers distal selon une fracture en languette (inférieure distale) dont l'origine est due à une flexion du support. Le bris est postérieur à l'usure du tranchant gauche, très discrètement ébréché et émoussé par la découpe d'une matière carnée ou cutanée tendre (fig. 14, photo 2).

Ce poignard de type pisciforme sur lame de NACAL peut être comparé à celui de la Motte-aux-Magnins (niveau ABC, Jura; Pétrequin dir., 1997), à certains éléments de Puyraveau et de Lavérré dans le Poitou (Patte, 1971) ou à celui de Chambretaud (Vendée; Ihuel, 2004).
- Poignard 2 (BP00-1001-05 et BP00-1001-08, fig. 9)

Ce silex noir uni rappelle notamment les gîtes de Larcy ou de Bossay-sur-Claise. La pièce mesure $210 \mathrm{~mm}$ pour $40,5 \mathrm{~mm}$ de large et $9,8 \mathrm{~mm}$ d'épaisseur maximale. La flèche est plutôt rectiligne. L'épaisseur est assez irrégulière et l'on observe de fortes ondulations en partie distale. Les négatifs de la face supérieure montrent qu'il s'agit d'une deuxième lame après un réépannelage partiel. Son profil indique un grand enlèvement en partie distale, masqué par le polissage de la face supérieure. L'agencement des enlèvements et l'irrégularité du support nous conduisent à rapprocher prudemment ce support d'une lame de NACAL.

Un polissage de la moitié distale a été effectué par une abrasion transversale oblique sur un matériau dur et grossier, comme en témoignent de profondes stries. Ce polissage important fût peut-être étendu à l'ensemble de la moitié distale dans un état primaire. La retouche de cette moitié distale, assez irrégulière et peu couvrante, a été effectuée par pression ou percussion tendre soignée. Une retouche semi-abrupte courte par percussion tendre se surajoute ensuite sur ces tranchants et affecte essentiellement le bord droit. Une troisième série d'aménagements se surimpose encore sur ce bord droit, sous la forme d'une microretouche qui donne un aspect microdenticulé au tranchant. Il s'agit probablement d'une opération d'entretien des tranchants, car elle recoupe, surtout à droite, un lustre de coupe de céréales (fig. 14, photo 3) discernable à l'œil nu. La moitié proximale est aménagée par une retouche bifaciale réalisée dans le but d'amincir la base. Sur la première face, les enlèvements larges sont obtenus par percussion tendre dans le sens senestre. Sur la deuxième surface, les enlèvements sont rasants à semi-abrupts et obtenus par pression ou percussion soignée, également dans le sens senestre.

Ce poignard est brisé en son milieu, exactement à la limite de la retouche bifaciale, très vraisemblablement au ras de l'emmanchement, comme le suggèrent non seulement la morphologie de l'objet, mais aussi la limite du lustre végétal et la différence d'état de surface entre les deux moitiés. La fracture est en languette supérieure distale.

Les poignards pisciformes polis attribuables à la production NACAL sont peu et mal documentés. On notera tout de même celui de Chambretaud en Vendée (Ihuel, 2004), ainsi que les nos 998.2.2 et 1269 de Puyraveau dans les Deux-Sèvres (Patte, 1971). Sur des éléments plus tardifs, le polissage intensif constitue un apport esthétique final à l'objet. Ce procédé est manifeste sur les lames exhumées aux Pays-Bas et à Jersey (Delcourt-Vlaeminck, 2004 ; Patton, 1995).

- Poignard 3 (BP00-1001-06, BP01-1010-02 et BP01-1010-04, fig. 10)

Le silex possède une teinte cire claire unie. Cette lame, qui mesure $180 \mathrm{~mm}$ de long pour $33 \mathrm{~mm}$ de large et 9,3 mm d'épaisseur maximale, n'a jamais été beaucoup plus longue, à peine $20-21 \mathrm{~cm}$, ce que révèlent les fortes ondulations en partie distale, associées à une épaisseur décroissante, ainsi que la 


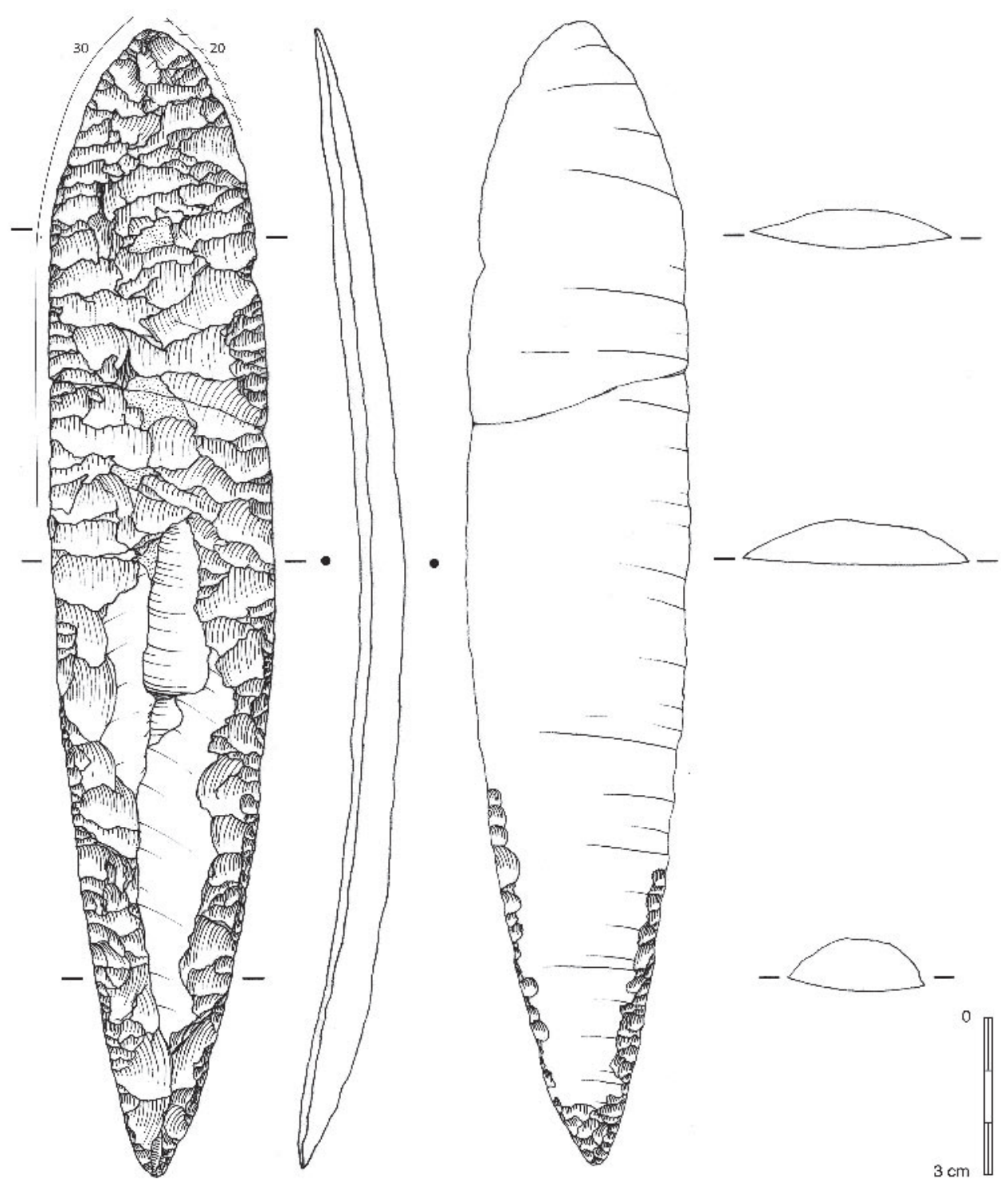

Fig. 9 - Poignard n ${ }^{\circ} 2$ (BP00-1001-05 et BP00-1001-08). Poignard en silex du Grand-Pressigny, de couleur brun noir tel le faciès siliceux de Larcy. La partie active de la lame a été préparée par polissage, antérieurement au façonnage par la retouche. La soie est préparée par une retouche bifaciale. Cassée à la limite de l'emmanchement, cette lame témoigne d'une utilisation sur des graminées antérieure à sa retouche.

présence d'une esquille bulbaire à la base de l'outil. Il s'agit d'une lame à deux pans. L'abondance des lancettes sur les deux négatifs près de la nervure centrale est l'indice d'un faible chevauchement des deux enlèvements. Ces caractères sont atypiques dans le cadre d'une production de livre de beurre et figureraient parmi un matériel de second choix, provenant peut-être de la reprise de ce type de nucléus. La production NACAL correspondrait mieux aux qualités de cette lame, mais elle n'y figurerait pas plus dans le premier choix.

Il s'agit d'un poignard unifacial. La moitié distale est retouchée par pression et la moitié proximale par percussion tendre pour réduire la largeur de la lame. Une dernière série de petits enlèvements par percussion tendre ou pression régularise les tranchants, qui sont parfaitement vifs et dont de minuscules denticulations accrochent encore le doigt. Ils sont cependant marqués, sur toute la moitié distale, par une discrète usure de découpe de matière tendre carnée ou cutanée. La même usure s'observe sur le côté droit de l'extrémité de la base du poignard, jusqu'au bout de laquelle se prolonge le tranchant, malgré l'épaississement du bord, grâce à une retouche écailleuse finement reprise. Ceci pourrait indiquer un usage hors manche, ou un manche n'emprisonnant pas l'extrémité proximale. Comme le poignard précédemment décrit, ce spécimen est cassé sous sa plus grande largeur, à la limite de l'usure du tranchant qui pourrait aussi correspondre à une limite d'emmanchement. Dans la moitié distale de la lame, les deux tiers de l'arête dorsale, là où elle est la plus saillante, ont été érodés (fig. 14, photo 4) par le contact prolongé ou répété avec un matériau souple abrasif (fourreau?), ce qui trahit une durée de vie de l'instrument plus longue que ce que suggère la faible usure finale des tranchants. 


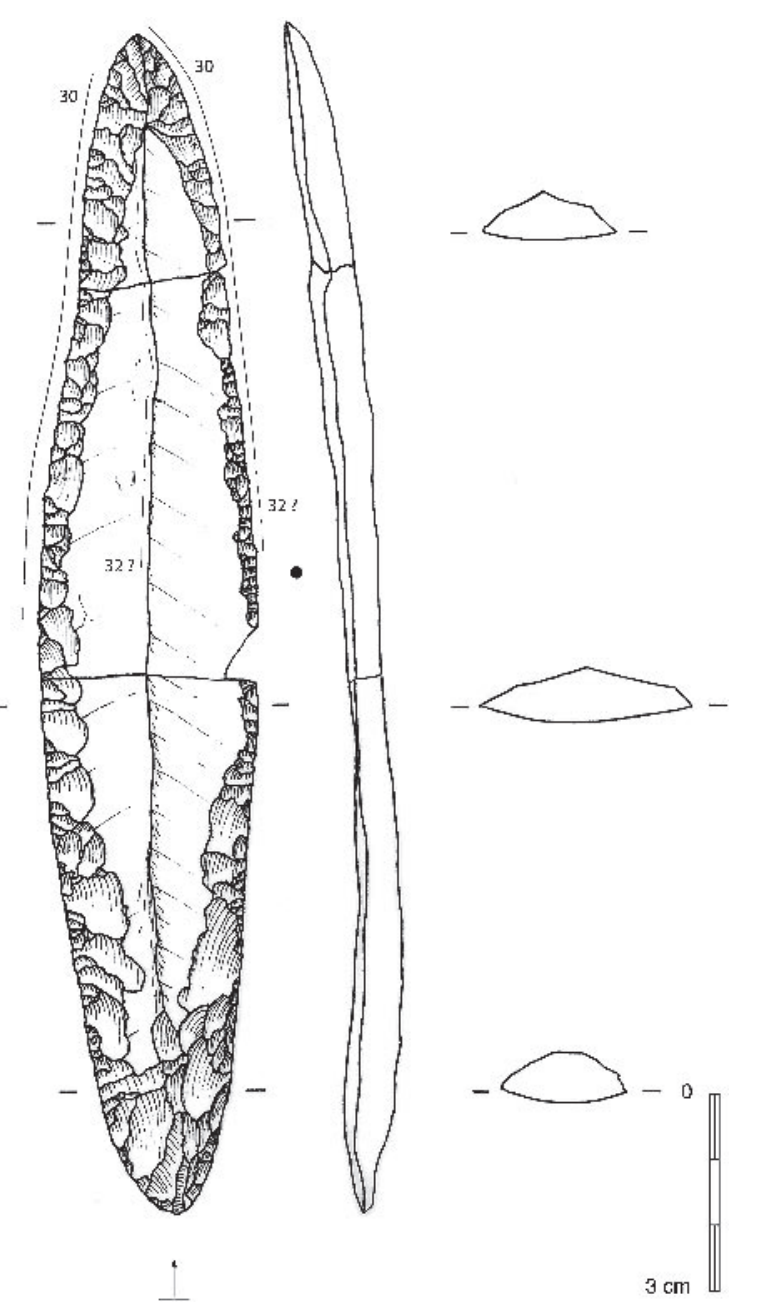

Fig. 10 - Poignard n ${ }^{\circ} 3$ (BP00-1001-06 et BP01-1010-02 et BP01-101004). Poignard en silex du Grand-Pressigny, de teinte cire, cassé en trois fragments. Il témoigne d'une utilisation sur de la matière tendre carnée ou cutanée. La cassure proximale, correspondant à une limite de patine différentielle ainsi qu'à la limite de la zone active, témoigne de la séparation entre partie emmanchée et partie active.

D'un point de vue typologique, cette lame est à rapprocher de certains éléments comme les $\mathrm{n}^{\text {os }} 6$ ou 8 de Fleuré, ou encore les poignards C, G et J de Puyraveau (Deux-Sèvres) à section préférentiellement triangulaire (Patte, 1971).

- Poignard n 4 (BP00-1001-04 et BP00-1001-09, fig. 11)

Le silex est brun clair, riche en quartz, et assez fortement translucide, ce qui pourrait le rapprocher notamment de faciès visibles à Bossay-sur-Claise. La lame mesure $223 \mathrm{~mm}$, sa largeur est de $35,5 \mathrm{~mm}$ et son épaisseur maximale est de $11,5 \mathrm{~mm}$. Sur la face supérieure, il est possible d'observer les vestiges d'un grand enlèvement laminaire près du bord droit. La majeure partie de la surface est constituée d'enlèvements transversaux, très plans. Les contre-bulbes indiquent clairement la proximité d'une crête. En partie proximale, l'un des derniers enlèvements laminaires sur le bord gauche efface une partie de cette crête et rebrousse en partie mésiale. Ces observations ne nous permettent pas de trancher avec assurance sur la méthode de débitage employée. D'un côté, l'épaisseur proximale importante, l'agencement convergent du débitage et les enlèvements transversaux plans plaident en faveur de la méthode NACAL. De l'autre, il est possible qu'une lame de livre de beurre débitée sur le flanc gauche du nucléus après le débitage partiel de la crête présente un aspect analogue, ainsi que cela a pu être documenté à la Claisière (Villes, 2004; Pelegrin et Ihuel, 2005). Il est toutefois étonnant que les négatifs de réépannelage ne soient pas plus étroits. Dans un cas comme dans l'autre, la lame n'a jamais été beaucoup plus longue que 23 à $24 \mathrm{~cm}$, ce qui reste compatible avec une toute dernière lame de livre de beurre, mais constitue alors un élément de second choix dans le plein débitage.

Le façonnage de la pointe est limité. Il est obtenu par pression et percussion sur les deux bords. La retouche est inexistante sur une petite plage du bord droit, ce qui témoigne de la grande «fraîcheur» du support, non réaffûté. La régularisation des bords est discontinue, effectuée par une retouche par percussion tendre qui ménage quelques zones brutes. Dans la partie proximale, cette retouche est précédée d'enlèvements semi-couvrants, creux, qui réduisent la largeur du support. La finition de la base est réalisée par pression, aménageant d'abord la face inférieure, puis supérieure. Bien que ne présentant pas de signe de ravivage, ce grand poignard a tout de même été utilisé, lui aussi, pour découper de la matière carnée ou cutanée résistante (fig. 14, photo 5). De même, il est cassé juste à la limite de l'usure de ses tranchants. Ce spécimen n'ayant pas été recollé après la fouille, les pans de cette fracture ont pu être examinés avec grand profit : elle apparaît ancienne, car la surface en est légèrement plus marquée que le fond d'une ébréchure récente, plus mate. Un autre indice d'ancienneté est la présence de filaments végétaux (microracines ?) observés sur toutes les pièces et jusque sur ces deux pans de fracture. Là encore, plusieurs arguments convergent pour suggérer un bris du poignard à la limite de son manche au moment de son dépôt.

La réduction de la base par retouche directe, la pointe en ogive étroite, l'utilisation importante de la percussion tendre et les bords subrectilignes sur les trois quarts de la pièce sont des éléments très comparables aux lames de Moigny, attribuées à une production issue de livres de beurre (Mallet et al., 1994).

- Le fragment de poignard 5 (BP01-1015-03, fig. $12 \mathrm{n}^{\circ} 4$ )

Il s'agit d'un silex brun sombre, de grain fin, translucide et très homogène. Il pourrait être rapproché des silex de la vallée du Brignon, dont le gisement le plus connu est Larcy, à Neuilly-le-Brignon (Aubry, 1991). Le fragment mesure $32 \mathrm{~mm}$ de long par $25 \mathrm{~mm}$ de large et $8 \mathrm{~mm}$ d'épaisseur. Le support laminaire présente deux enlèvements transversaux provenant de gauche, ce qui est bien insuffisant pour l'attribuer à l'une ou l'autre méthode de débitage (livre de beurre ou NACAL). Les deux cassures sont de type languette supérieure, avec des indices de fracture radiale qui témoignent d'un choc violent. Quatre générations de 


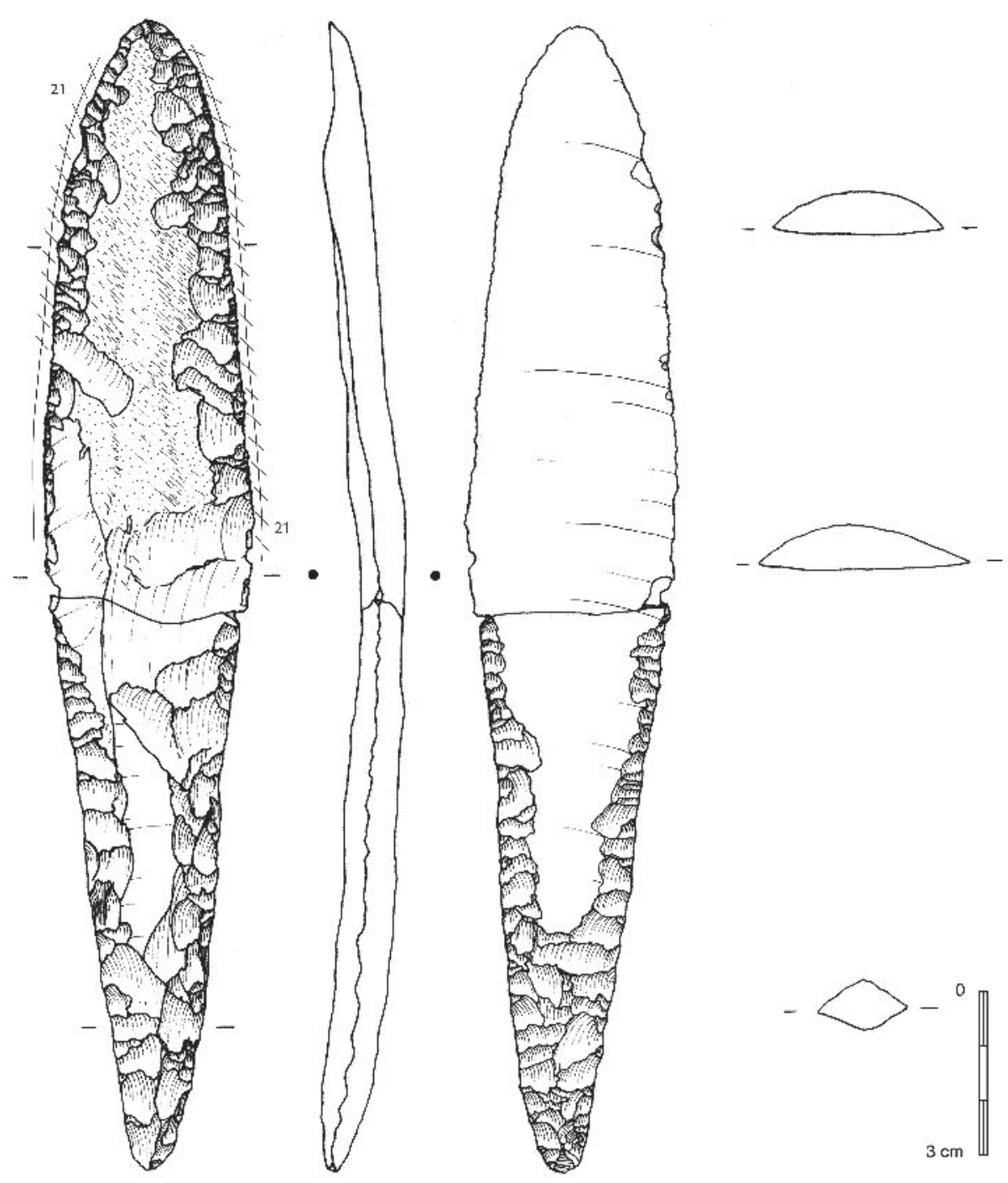

Fig. 11 - Poignard n 4 (BP00-1001-04 et BP00-1001-09). Poignard en silex du Grand-Pressigny, cassé en deux par flexion à la limite probable de l'emmanchement. L'analyse tracéologique témoigne d'une utilisation pour la découpe d'une matière carnée ou cutanée résistante.

retouches sont visibles. La première est semi-couvrante, rasante et semble effectuée à la pression. Les trois suivantes sont irrégulières, semi-abruptes à abruptes et sont réalisées par percussion directe, vraisemblablement dure. La dernière génération aménage la fracture et témoigne du réemploi du fragment dont la surface est microscopiquement très marquée, probablement à force de manipulations. Une usure de raclage rasant d'une matière souple abrasive est discernable sur un centimètre et demi de son bord gauche.

- Le fragment apical de poignard 6 (surface)

Le silex est cire clair; on peut y observer un grand fragment de bryozoaire. La pièce mesure 40 x 22 x $7 \mathrm{~mm}$. La face supérieure est le négatif d'un enlèvement transversal provenant de droite, antérieur au débitage. La retouche semi-abrupte est réalisée par pression et localement des enlèvements courts par percussion sont visibles. Elle recoupe partiellement un lustre de coupe de céréales (fig. 14, photo 6).
- Le fragment retouché de lame, utilisé en briquet (BP01-1010-06, fig. 12, n 6)

Le silex est de couleur cire et se rapporte à un faciès classique de la région du Grand-Pressigny. C'est un fragment proximal de lame qui mesure 79 x 29,5 x $14 \mathrm{~mm}$. Un reste de talon est conservé, bien que fortement altéré par une utilisation comme briquet (fig. 14, photo 7). Sur la face supérieure, un enlèvement laminaire est postérieur à l'épannelage provenant de gauche. La pièce présente une fracture en nacelle qui s'est opérée lors du débitage, trahissant ainsi l'usage de la percussion indirecte. Il s'agit vraisemblablement d'une lame de livre de beurre ou de reprise, cassée au débitage. L'ensemble de la pièce a été aménagé par des enlèvements irréguliers abrupts, souvent clactoniens, obtenus par percussion directe dure. Une petite plage sur le bord gauche fait exception : elle est très soigneusement retouchée par percussion dans un dernier cycle d'aménagement. Elle délimite une zone active, marquée par le raclage d'une matière végétale fraîche dont 

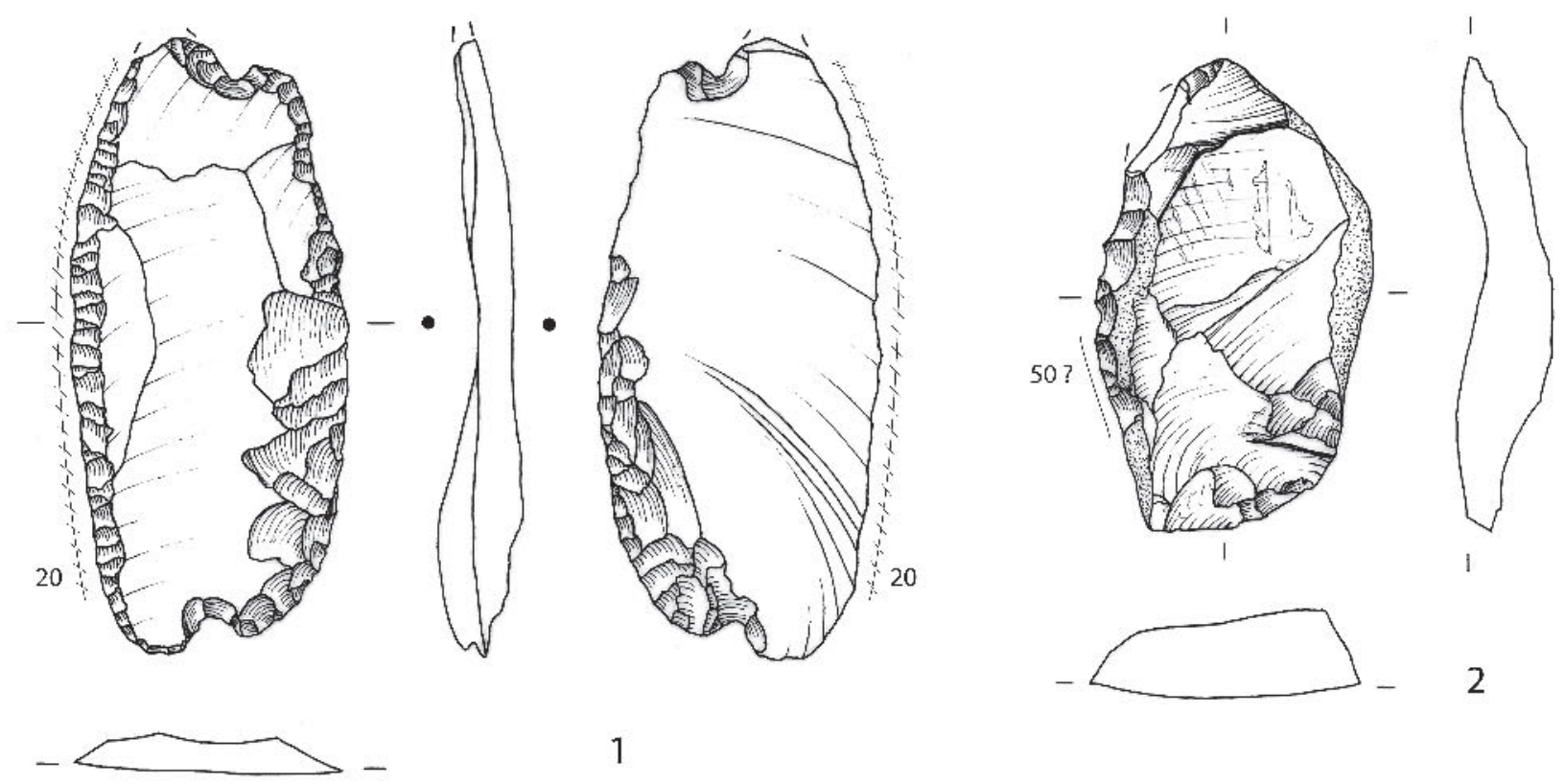

1
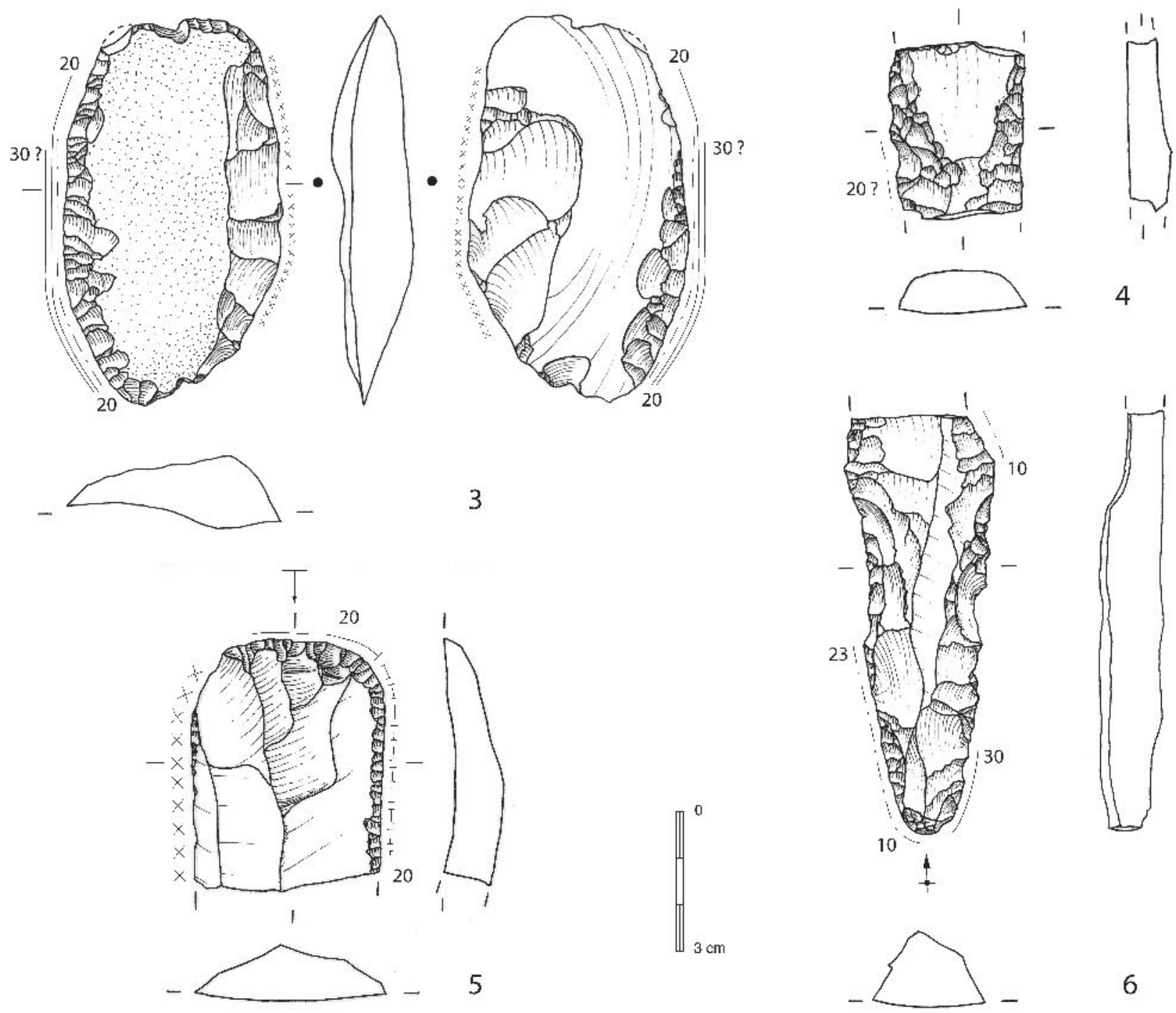

Fig. 12 - Outillage en silex du Grand-Pressigny, hormis le $n^{\circ} 5$ provenant d'Aquitaine. $\mathrm{N}^{\circ \mathrm{s}} 1$ et $3:$ scies à encoches; $n^{\circ} 2:$ racloir sur éclat; $n^{\text {os }} 4$ et 6 : fragments de lames de poignards, le $n^{\circ} 6$ ayant été utilisée en briquet; $n^{\circ} 5$ : fragment proximal de lame large retouchée. 
la signature est très particulière (poli 23; fig. 14, photo 8) et qui pourrait correspondre à l'étêtage de céréales, selon les expériences de P. Anderson (Beugnier et Plisson, 2004). Le talon présente des esquilles, des traces de percussion violente et un émoussé qui suggèrent fortement un usage en briquet. Deux autres petites zones actives sont présentes, relatives au raclage d'une matière minérale et à la découpe d'une matière tendre abrasive. Toute la surface de l'instrument est marquée, vraisemblablement par les manipulations et le transport. Le fonctionnement en briquet, du reste, génère une poussière abrasive qui imprègne les doigts et lustre les éléments tenus (Collin et al., 1991).

L'objet décrit illustre ainsi un cas de recyclage d'un «raté de débitage» qui n'est pas si opportuniste qu'il pourrait paraître de prime abord, puisqu'il s'inscrit dans le cycle de vie probablement codifié des lames pressigniennes et correspond précisément au stade ultime de leur consommation (Beugnier et Plisson, 2004). Le transport à longue distance d'un tel fragment avait donc du sens.

- La scie à encoches $\mathbf{n}^{\circ} \mathbf{1}$ (BP00-1001-01, fig. 12, $\mathrm{n}^{\circ} 3$ )

Le matériau est un silex gris, de grain fin, au cortex mince et résistant à l'ongle. L'éclat cortical est court et large, il mesure $73 \mathrm{~mm}$ de long par $41 \mathrm{~mm}$ de large et $14 \mathrm{~mm}$ d'épaisseur. Le bord droit peut être considéré comme un dos, puisque aménagé par un façonnage bifacial et volontairement émoussé. Le bord gauche est façonné par une retouche inverse rasante très soignée, puis par une retouche directe semi-abrupte assez régulière, obtenue par pression ou percussion tendre. $\mathrm{Ce}$ tranchant a servi à couper et fendre un végétal rigide et, peut-être, à couper une matière carnée ou cutanée. Les coches sont chacune aménagées par un enlèvement clactonien, mais ne présentent aucune trace d'usage. En revanche, les parties hautes du volume de l'objet sont marquées, vraisemblablement par la préhension.

- La scie à encoches $\mathbf{n}^{\circ} 2$ (BP00-1001-13, fig. 12, $\left.\mathrm{n}^{\circ} 1\right)$

Le silex est cire clair, faciès classique de la région du Grand-Pressigny. Le support est un éclat d'épannelage de livre de beurre, de la deuxième série. Il mesure $85 \mathrm{~mm}$ de long et $39 \mathrm{~mm}$ de large pour une épaisseur maximale de $11 \mathrm{~mm}$. L'éclat a d'abord été fractionné par flexion en partie distale. Puis le bord droit a été façonné par des enlèvements larges et irréguliers, d'abord directs, ensuite inverses. Le bord gauche a été réalisé par une retouche directe, courte et régulière, par percussion tendre ou pression, qui recoupe un stade antérieur de fonctionnement comme couteau sur un végétal relativement rigide. Les coches sont réalisées par des enlèvements bifaciaux et sont vierges de traces.

\section{- Le fragment de lame à aménagement proximal} (BP00-1001-03, fig. 12, n 5)

Cette pièce est en silex brun sombre, provenant probablement de niveaux du Jurassique aquitain (examen N. Mallet et P. Fouéré). L'objet mesure $46 \mathrm{~mm}$ de long pour $37 \mathrm{~mm}$ de large et $10 \mathrm{~mm}$ d'épaisseur. Bien que fracturé, le support présente une flèche importante. Il s'agit d'une lame à trois pans dont l'agencement des enlèvements est 3-1-2. La troncature proximale est réalisée par quatre larges enlèvements débités en percussion tendre. Une petite retouche courte très régulière aménage le bord droit et la partie proximale, tandis que le bord gauche semble volontairement poli pour réaliser un dos. Ces différents aménagements se rapprochent des outils du type scies à encoches. Finalement, un réaffutage très léger, par micro-enlèvements, a été opéré sur le bord droit, recoupant un lustre relatif à la coupe d'un végétal relativement rigide.

L'industrie lithique taillée comporte encore trois pointes de flèches à pédoncule et ailerons bien dégagés, plus ou moins élancées (fig. 13). Ces types de forme sont bien attestés dans le Néolithique récent et final régional, comme à Diconche (Fouéré, 1994; Burnez et Fouéré dir., 1999) et à Challignac (Louboutin et al., 1997) en Charente, ou comme aux Vaux et au Camp des Châtelliers, deux gisements de la commune de Moulins-surSéphons dans l'Indre (Hamon, 1999). Aucune des trois pointes ne semble neuve. La plus grande (fig. $13, \mathrm{n}^{\circ} 1$ ) montre un microflutage de son apex, tel qu'il peut s'en produire au fond d'un carquois ou lors d'un tir dans une substance tendre. Celle de dimension moyenne (fig. 13 $\mathrm{n}^{\circ} 3$ ) a l'extrémité de ses ailerons volontairement émoussée, comme cela a déjà été observé sur des pointes usagées (Gassin, 1996), ce qui indique un spécimen en
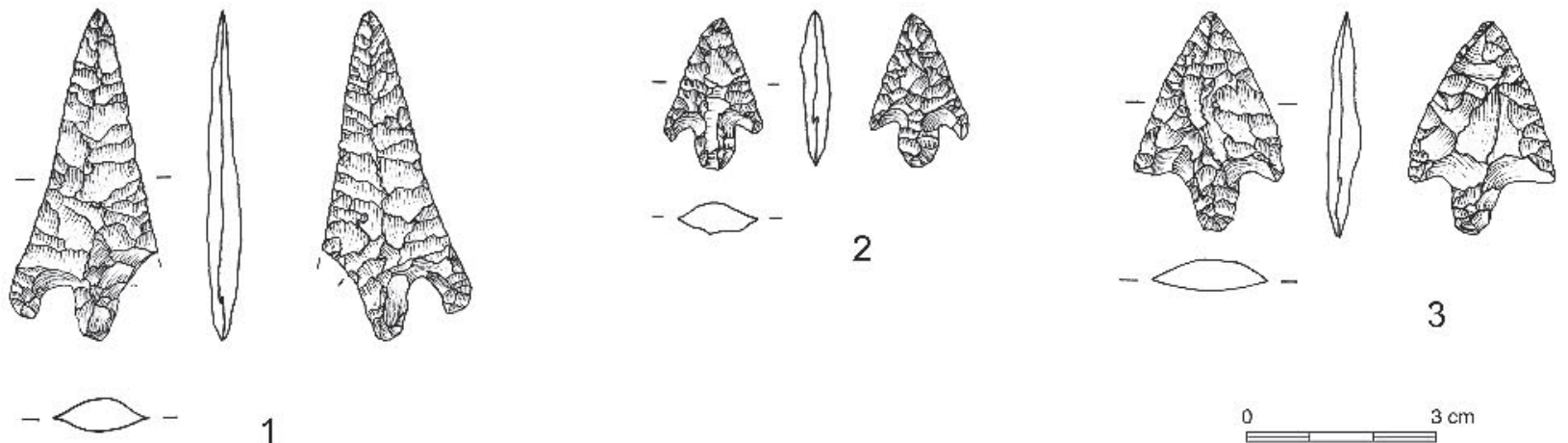

3

Fig. 13 - Pointes de flèches à pédoncule et ailerons, dont l'examen tracéologique témoigne qu'elles ont probablement été tirées, ou qu'elles étaient tout au moins en condition de fonctionnement. 

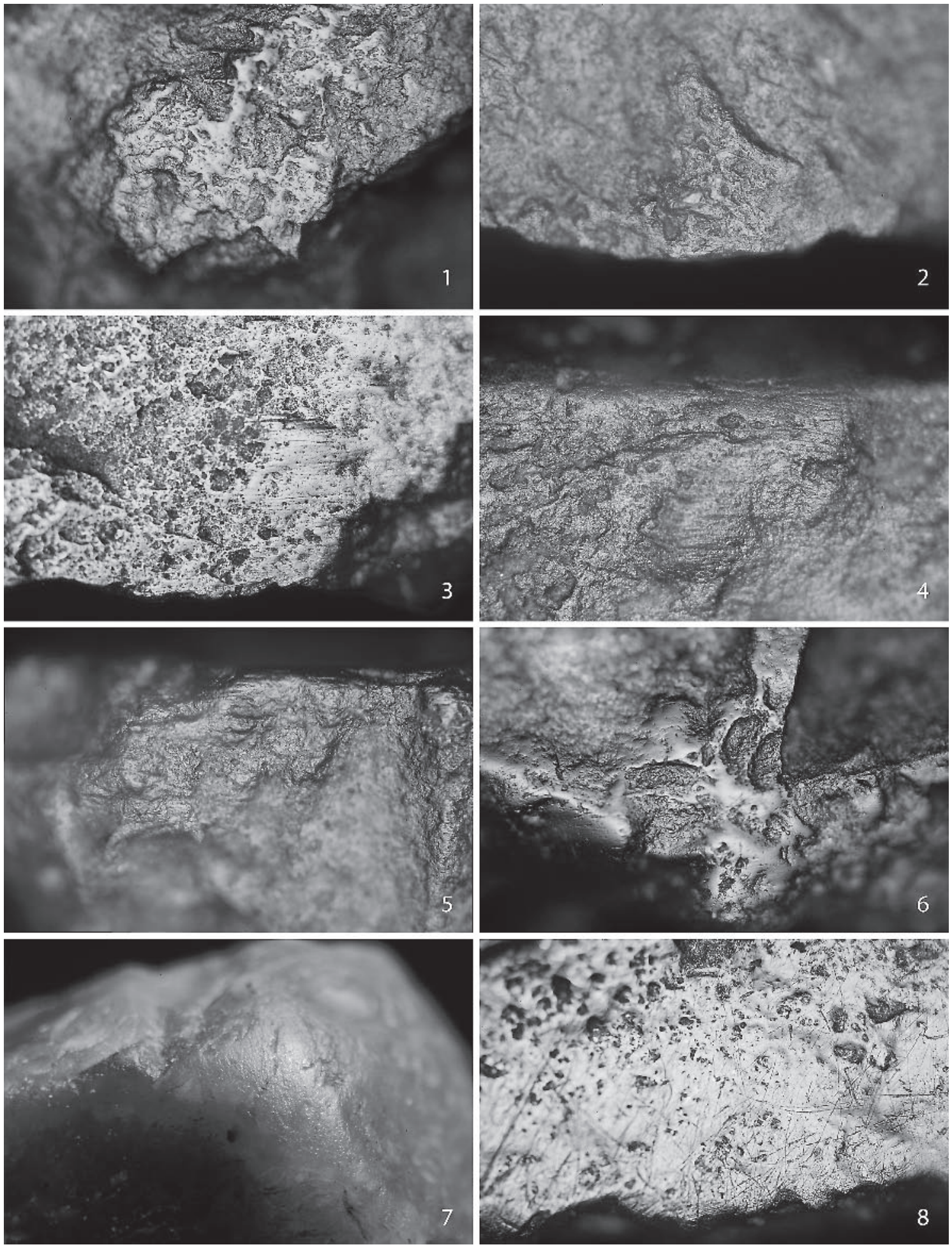

Fig. 14 - Photo 1 - Poignard n 1 (BP00-1001-10 et BP00-1001-11), détail de l'usure à l'apex, bord droit, face inférieure, recoupée par la retouche, attribuable à la coupe de végétaux. 200 x. Photo 2 - Poignard n 1 (BP00-1001-10 et BP00-1001-11), détail de l'usure, bord gauche, moitié distale sous la cassure, face inférieure, attribuable à la découpe d'une matière carnée ou cutanée tendre. $200 \mathrm{x}$. Photo 3 - Poignard n 2 (BP00-1001-05 et BP00-1001-08), détail de l'usure, bord gauche, partie médiane de la moitié distale, face inférieure, recoupée par la retouche, attribuable à la coupe de céréales. 100 x. Photo 4- Poignard nº 3 (BP00-1001-06 et BP01-101002 et BP01-1010-04). Détail de l'usure de l'arête dorsale, moitié distale, marquée par le frottement d'une matière souple abrasive. $100 \mathrm{x}$ Photo 5 - Poignard n 4 (BP00-1001-04 et BP00-1001-09). Détail de l'usure, bord gauche, proche de l'apex, face supérieure, attribuable à la découpe de matière carnée ou cutanée résistante. 200 x. Photo 6 - Fragment apical de poignard 6 (surface). Détail de l'usure, bord droit, face inférieure, proche de la fracture proximale, attribuable à la coupe de céréales. 200 x. Photo 7 - Fragment de poignard (BP01-1010-06). Détail de l'émoussé macroscopique de l'extrémité proximale, attribué à un usage comme briquet. 15 x. Photo 8 - Fragment de poignard (BP01-1010-06). Détail de l'usure, bord gauche, face inférieure, moitié proximale. 200 x. 
condition de fonctionnement. La plus petite (fig. 13, $\mathrm{n}^{\circ}$ 2) présente quelques irrégularités de tranchant qui trahissent soit une finition imprécise, soit des microendommagements d'impact.

\section{Les éléments de parure}

La parure est représentée par onze perles et fragments (fig. 15). Ce sont toutes des perles discoïdes en lignite. Si leur diamètre semble standardisé aux alentours de $8 \mathrm{~mm}$, leur épaisseur varie du simple $(2 \mathrm{~mm})$ au triple $(6 \mathrm{~mm})$. Les perforations, généralement bien cylindriques, montrent deux types d'orientation dans l'axe de la perle. Les perles les plus plates portent une perforation généralement bien perpendiculaire à la section. Les plus épaisses montrent, elles, des perforations obliques. Elles n'ont été retrouvées qu'au sein de la fosse FS 003.

Du point de vue de la matière première, toutes sont en lignite, ou «jayet». Notons qu'une origine lointaine (Midi-Pyrénées ou Bretagne, Barge, 1982; Vergély, 2001 et 2002) n'est pas la seule hypothèse possible. C. Chevillot signalait ainsi la présence géologique de ce matériau dans la région de Rochechouart (soit à environ $25 \mathrm{~km}$ au nord-ouest), à la base du Cénomanien (Chevillot, 1976). De plus, le même auteur décrit également la présence d'ateliers de fabrication de bracelets
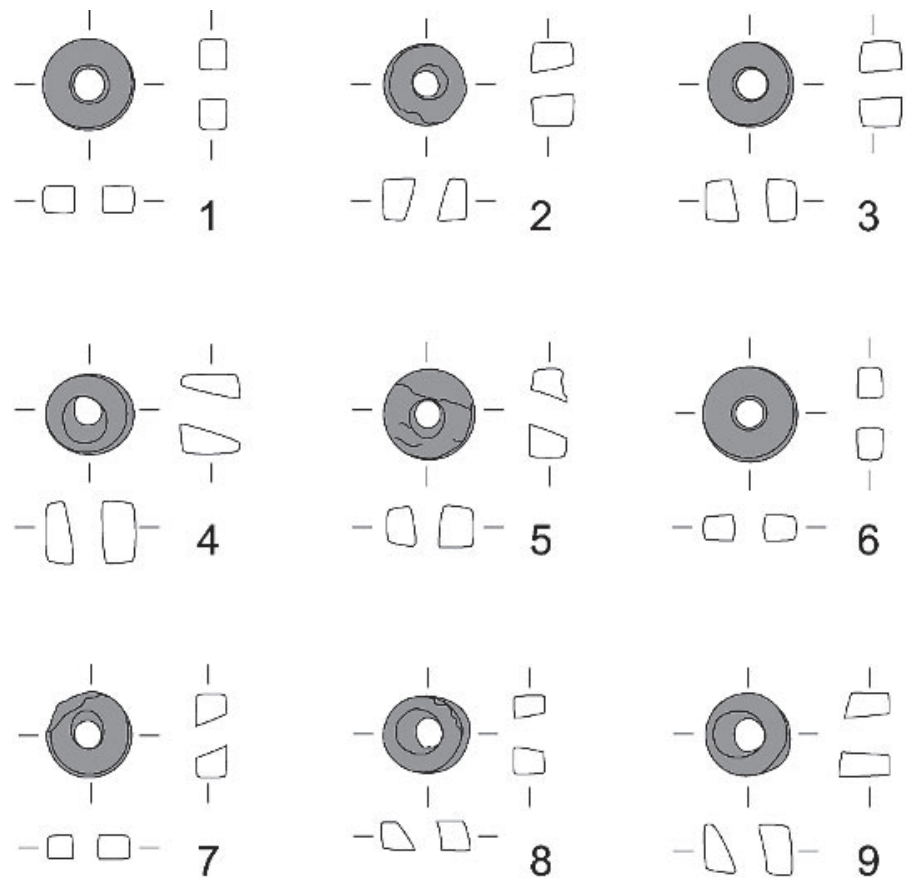

8
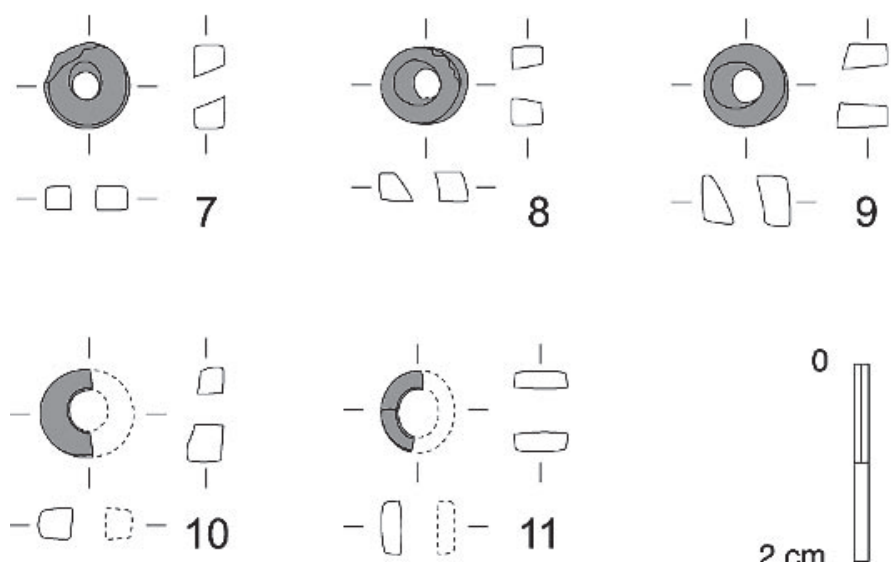

0

$2 \mathrm{~cm}$ en lignite, datés de l'Âge du Fer, à une trentaine de kilomètres à l'est du Bois Pargas (ibid.). La proximité géographique de ces sources et de cet atelier plaide dans le sens d'une possible origine régionale de ce matériau.

\section{INTERPRÉTATION FONCTIONNELLE DU SITE}

\section{Un site funéraire?}

Malgré l'absence d'ossements, le site peut probablement être compris comme une sépulture collective monumentale, en raison de sa structure, de sa localisation et de son mobilier. L'hypothèse d'une cache d'objets est une interprétation qui a également été prise en considération. Cependant, les cas connus de dépôts ou de cachettes de lames pressigniennes ne rassemblent habituellement qu'un type unique de vestige : des lames brutes. Citons par exemple le célèbre dépôt de la Creusette au Barrou (Indre-et-Loire; Geslin et al., 1982) qui rassemblait 133 lames brutes, ou, sur la même commune, celui des Ayez qui réunissait lui 122 lames brutes (Cordier, 1986; Marquet et MilletRichard, 2000). Les dépôts de lames retouchées en poignards sont extrêmement rares, puisqu' on ne peut guère citer que ceux de Moigny (Mallet et al., 1994) ou de la forêt de Damps (Cordier, 1986). Ces deux gisements sont situés à près de $200 \mathrm{~km}$ au nord du Grand-Pressigny. Les autres dépôts potentiels de lames de poignards ne rassemblent que deux ou trois pièces, comme Boutigny (ibid.), et leurs contextes archéologiques sont tels qu'on ne peut exclure l'hypothèse qu'il s'agisse là de sépultures non reconnues. Quoi qu'il en soit, les dépôts ne comportent que des lames de poignards seules ou, au mieux, associées à des grattoirs sur lames. Ces éléments sont toujours très stéréotypés et ne montrent aucune évidence d'utilisation. Par contraste, la présence au Bois Pargas, au sein d'une même fosse, de poignards façonnés, qui ne sont plus tout à fait neufs, associés à des armatures usagées, de la céramique et des parures, évoque plutôt un mobilier funéraire, probablement relatif à plusieurs défunts à en juger par le nombre de pièces. Nous sommes ici en présence d'un ensemble que l'on retrouve de façon classique dans les dolmens durant le troisième millénaire en Europe de l'Ouest continentale (cf. par exemple Patte, 1971; Joussaume, 1977; Vuaillat, 1998). Un tel viatique montre que la communauté fréquentant le site se situait à l'intérieur de réseaux complexes et géographiquement étendus : les poignards pressigniens indiquent des connexions vers le nord à plus de $150 \mathrm{~km}$, tandis que le fragment de poignard en silex jurassique témoigne de relations avec le monde aquitain (Delage, 1993; Fouéré, 1994).

La fraîcheur des trois scies à encoches va également dans le sens d'un dépôt intentionnel sans doute d'ordre funéraire. Mais ajoutons qu'à notre connaissance très peu de scies à encoches ont été découvertes dans de tels contextes. L'exemplaire unique trouvé dans la grotte du Gardon (Ambérieu-en-Bugey, Ain) est attribué

Fig. 15 - Perles en jayet provenant de l'US 1010 et de la souche 1001. 
à un niveau d'occupation domestique (Perrin, 2003). Le même constat doit être renouvelé à Bilgroix (Arzon, Morbihan), l'outil étant situé à quelques mètres de la tombe dans un contexte lui aussi domestique (Lecornec, 1996). C'est également le cas de tous les exemplaires signalés dans les stations lacustres jurassiennes et alpines (Mallet, 1992). La seule évidence de scie à encoches emportée dans la tombe est celle de l'allée sépulcrale de l'usine Vivez à Argenteuil (Mauduit et al., 1977). Le cas existe donc, mais reste une exception dans la documentation. De même, il est régionalement très rare de pouvoir documenter un cas d'expression funéraire non mégalithique pour cette période, puisque les sépultures limousines du Néolithique final ne sont guère connues que par des dolmens (Vuaillat, 1998).

\section{Des lames brisées intentionnellement?}

Toutes les lames de poignards exhumées de la fosse FS 003 étaient brisées. Ce constat suscite une interrogation sur les circonstances de leur fracturation : sontelles la conséquence de leur utilisation, la matérialisation d'un acte volontaire (la cassure du briquet n'a pas d'importance dans cette interrogation, puisque la lame a vraisemblablement été acquise sous cette forme, et qu'elle constitue certainement à ce titre un effet personnel d'un défunt) ou l'effet des bouleversements ayant affecté le gisement?

Premier constat, ce bris touche seulement les poignards. Six fractures sur huit sont de morphologie en languette courte, révélatrices d'une rupture par flexion transversale du matériau et non par flambage. Ces cassures interviennent six fois sur huit en partie mésiale, dont trois fois en limite manifeste d'emmanchement (poignards 2 à 4). Un deuxième type de cassure est en languette associée à une fracturation radiale partielle, signe de forte énergie, vraisemblablement portée par un coup violent; il concerne un court fragment de poignard recyclé (poignard $n^{\circ} 5$ : fig. 12, $n^{\circ} 4$ ).

Le raccord in situ des fragments, l'antériorité des usures, leur attribution à des actions mécaniquement peu contraignantes, le faible degré de consommation des objets, des limites de cassures vraisemblablement à ras du manche et l'ancienneté tracéologique d'une fracture analysable sont autant d'indices convergents pour supposer un bris volontaire des poignards au moment de leur dépôt, puisque postérieur à l'usage et antérieur à la décomposition des matériaux organiques constituant les manches. Nous sommes peut-être là en présence d'un aspect du rituel funéraire. La nature des objets concernés n'est pas indifférente, puisque leur finalité dépassait nettement le champ technique et s'inscrivait dans une construction sociale et symbolique au travers de laquelle s'exprimait la compétition entre les hommes (Pétrequin et Pétrequin, 1988; Beugnier et Plisson, 2004). On peut se demander alors si la signification funéraire résidait dans leur choix ou dans leur traitement. S'agissait-il de dépôts marquant le caractère sépulcral du lieu, comme les haches laissées à l'unité (Masset, 1997), ou bien de viatiques personnels, soit possession des inhumés, soit rappel de leur position sociale ? Leur bris plaide plutôt en faveur de l'hypothèse d'attributs personnels, comme leur association avec des pointes de flèches, un autre symbole masculin (Pétrequin et Pétrequin, 1990), qui n'est pas rare en milieu funéraire. Peut-être convenait-il ici de «tuer» cette représentation du défunt, ou plus simplement d'en prévenir le vol?

\section{CHRONOLOGIE ET DATATION}

Malgré l'absence de conservation des éléments organiques qui a empêché toute datation absolue du gisement, on peut proposer une attribution chronoculturelle au Néolithique récent ou final, soit grosso modo au troisième millénaire avant notre ère. C'est en tout cas ce que suggèrent l'aspect général de la céramique, celui de plusieurs poignards en silex pressigniens, ainsi que des scies à encoches ou des pointes de flèches à pédoncule et ailerons (Chevillot, 1989; Fouéré, 1994; Joussaume dir., 1998).

Régionalement, si les pointes de flèches à pédoncule et ailerons bien dégagés paraissent relativement courantes (Perrin, 2000), les poignards en silex sont plus rares. Ils sont cependant attestés dans la littérature au dolmen du Puychaud (Blond; Delporte, 1966), aux Forges à Saint-Cernin-de-Larche (ibid.), au dolmen de Maurioux dans la Creuse (Tixier, 1977), aux Zottes (Saint-Maurice-la-Souterraine, ibid.), au dolmen de Chez Boucher à la Croix-sur-Gartempe (Beausoleil et Picard, 1987), ou encore à Marsac (Joussaume et al., 2002). Tous ces gisements sont attribués à la culture d'Artenac, expression régionale du Néolithique final qui s'étend sur à peu près l'ensemble du troisième millénaire.

Cependant, la présence au Bois Pargas d'une forme à ailerons récurrents (fig. $13, \mathrm{n}^{\circ} 1$ ) pourrait suggérer une datation assez tardive au sein de cette vaste fourchette. Les autres formes d'armatures à pédoncule et ailerons sont par contre plus ubiquistes, puisque «dans la chronologie observée à Diconche (Burnez et Fouéré dir., 1999), [elles sont] présentes tout au long du troisième millénaire» (Joussaume et al., 2002, p. 63). Les scies à encoches en silex du Grand-Pressigny sont généralement elles aussi considérées comme tardives. Des travaux récents indiquent ainsi qu'elles se rattachent plutôt à la deuxième phase artenacienne, considérée comme contemporaine (Artenac IIa) ou postérieure (Artenac IIb) au Campaniforme, et qui se situerait chronologiquement dans la seconde moitié du troisième millénaire (Fouéré, 1994; Burnez et al., 1998; Burnez et Fouéré dir., 1999). Il faut cependant fortement moduler cette hypothèse chronotypologique, comme le signalait d'ailleurs N. Mallet dès 1992 (Mallet, 1992, p. 183), en se rappelant que, dans l'Est de la France comme en Suisse, les scies à encoches en silex du Grand-Pressigny sont plus largement attestées dans la première moitié du troisième millénaire que dans la seconde! C'est ainsi le cas avec la pièce provenant de la couche 37 de la grotte du Gardon dans l'Ain, attribuée au groupe de Chalain et datée entre 2900 et 2500 avant notre ère (Perrin, 2003, p. 292, sq.). C'est 
également le cas à Charavines, à Vinelz ou à Auvernier, où elles sont là aussi attestées dans des niveaux datés par dendrochronologie de la première moitié du troisième millénaire (Mallet, 1992) et plus précisément même entre 2700 et 2500 avant notre ère.

De façon générale, la présence de silex du GrandPressigny au Bois Pargas s'insère dans les processus généraux de diffusion de ces matériaux. Or, toutes les recherches récentes montrent que l'exportation de ces objets en silex du Grand-Pressigny ont eu lieu, pour l'essentiel, entre 2870 et 2450 avant notre ère (Mallet, 1992; Delcourt-Vlaeminck, 2002; Honegger, 2006), d'après la datation des gisements lacustres alpins.

Il est possible d'aller plus loin encore en revenant plus précisément sur les poignards eux-mêmes et sur une des scies à encoches aménagée à partir d'un éclat d'épannelage de nucléus. Il a récemment été mis en évidence deux phases d'exploitation des productions laminaires pressigniennes au Néolithique final. À côté des célèbres débitages sur livres de beurre, une nouvelle méthode de production a été identifiée, celle sur nucléus à crêtes antéro-latérales (ou «NACAL») : «Ce sont des nucléus à lames assez longues, plutôt larges et minces, de forme rectangulaire avec crête(s) et néocrêtes en position antéro-latérale tôt et répétitivement enlevées sous forme de lames débordantes, avec plan de frappe opposé d'aménagement ou de débitage » (Pelegrin et Ihuel, 2005, p. 46). Ce modèle a pu être défini par le jeu croisé d'études des modalités de production à partir des nucléus comme à Ligueil (Indre-et-Loire; Villes, 2003) et à partir d'une catégorie de grandes lames découvertes principalement en contexte funéraire dans l'Ouest de la France, travail initié par J. Pelegrin, puis mené conjointement avec l'un d'entre nous dans le cadre d'une thèse (Pelegrin, à paraître; Ihuel et Pelegrin, à paraître; Pelegrin et Ihuel, 2005; Ihuel, 2002). Malgré la réelle difficulté de corréler les données tirées de contextes aux fiabilités diverses et fortement éloignés les uns des autres, il nous paraît possible de distinguer un type ancien (NACAL) et un type récent (livres de beurre) de production de grandes lames, reconnus dans le secteur des ateliers tourangeaux.

\section{Les lames anciennes}

La datation du type ancien (NACAL) est assurée par trois gisements principaux. Il s'agit notamment des niveaux ABC de la Motte-aux-Magnins (Clairvaux, Jura) datés de 2980 av. J.-C. par dendrochronologie et attribués au style Clairvaux ancien, ainsi que de l'enceinte à céramique de style Kerugou de la Chevetelière (Saint-Mathurin, Vendée), datée par une trentaine d'échantillons de charbon entre 3100 et 2850 av. J.-C. Le gisement de Bilgroix, dont la fréquentation principale est contemporaine, fournit trois dates fiables réalisées sur des foyers entre 3150 et 2900 av. J.-C., associées également à du mobilier Kerugou (Lavier, 1996; Lecornec, 1996; Beugnier, 1997; Plisson et Beugnier, 2004 ; Viellet, 2005 ; Péridy, 1999 et 2005). Le recensement, non exhaustif, des sites impliquant cette production s'élève à 81 gisements dont 62 ont pu être étudiés à présent dans le cadre d'une thèse en cours (E. Ihuel).

\section{Les lames récentes}

Les premières lames que l'on peut attribuer avec certitude à la méthode livre de beurre sont celles des niveaux Lüscherz récent de Portalban «Terrain des sports », datés de 2787-2701 av. J.-C. Il est particulièrement notable de souligner qu'elles ne sont pas accompagnées de lames de la tradition précédente (NACAL). La situation est identique à Vinelz, Haufenaufahrt, pour les mêmes niveaux datés de 2760-2690 av. J.-C. ou encore pour le site du Bain des Dames à Saint-Blaise pour des dates similaires (Mallet, 1992; Honegger, 2001). Dans le niveau Lüscherz ancien 2A de Delley-Portalban II, rien ne permet d'affirmer parmi les dessins publiés qu'il s'agit bien là de lames issues de livres de beurre (ibid.). À Charavines, deux poignards à talon lisse pourraient peut-être évoquer des types anciens, mais ils pourraient tout aussi bien être reliés à des productions de nucléus plat (Mallet, 1992). En définitive, dès les niveaux d'occupation les plus anciens attribués à la consommation de lames produites sur livres de beurre, les lames de nucléus à crêtes antéro-latérales (NACAL) semblent avoir disparu, et ceci dès le début du XXVIII ${ }^{\mathrm{e}}$ siècle.

Ces deux productions ( $\mathrm{NACAL}$ » et «livres de beurre») sont donc exclusives l'une de l'autre au sein des réseaux d'exportations. À cet égard, leur reconnaissance et leur identification peuvent constituer de bons indices chronologiques. Cela est notamment vrai pour les sites consommateurs lointains et liés à des niveaux d'habitats. La situation est par contre beaucoup moins claire dans le domaine funéraire. Chacun des deux types de lames est en effet attesté dans des sépulcres distincts, mais nombreuses sont également les sépultures mêlant les deux productions. Dans le CentreOuest, c'est le cas du petit dolmen de Laverré à Aslonne (Vienne; Patte, 1971), de la sépulture mégalithique de Puyraveau à Saint-Léger-de-Montbrun (Deux-Sèvres ; ibid.), de la sépulture en fosse de Soccouteaux à Fleuré (Deux-Sèvres; ibid.), ou encore du monument funéraire de la Pierre-Virante à Xanthon-Chassenon (Vendée; Joussaume, 1977). Cette remarque est tout aussi vraie au nord de la Loire, comme dans le dolmen de Vilaine à Sublaines (Loir-et-Cher; Cordier et al., 1972) ou dans la sépulture d'Éteauville à Lutz-en-Dunois (Eure-et-Loir; Nouel et al., 1965). Dans toutes ces sépultures, le mobilier associé est attribué au Néolithique récent ou au Néolithique final. Parmi elles, certaines sont en fosse, semi-enterrées, sous dalle ou sous petit tumulus avec ou sans construction semimégalithique comme la sépulture de Soccouteaux, celle de Vilaine ou encore celle d'Éteauville, ce que G. Bailloud regroupait sous un même groupe lors de l'étude de la sépulture d'Éteauville (ibid., p. 610).

Deux hypothèses peuvent rendre compte du fonctionnement et de la datation de la sépulture du Bois Pargas. Soit il s'agit d'un site sépulcral ayant connu 
deux phases d'utilisation principales, l'une contemporaine des exportations de productions NACAL (soit entre 3100 et 2800 av. J.-C. grosso modo), l'autre contemporaine des productions sur livres de beurre (soit entre 2800 et 2450 av. J.-C. environ). Cette double utilisation de la sépulture n'exclut d'ailleurs pas une continuité dans l'occupation du site. Soit il s'agit d'un gisement n'ayant connu qu'une seule fréquentation, contemporaine des productions sur livres de beurre, mais où certains objets furent acquis bien longtemps avant d'être déposés dans la structure funéraire. Seule l'investigation complète du plateau sur lequel se situe le site pourrait fournir des arguments directs pour répondre à cette question, si tant est que d'autres structures soient conservées, ce qui est loin d'être évident. En l'état des connaissances, il est délicat de trancher entre les deux interprétations. On peut simplement noter que le faible degré d'usure des pièces de la première période plaide plutôt en faveur d'une fréquentation longue ou répétée de la sépulture entre 3100 et 2450 avant notre ère environ, conformément à une tendance déjà soulignée (Pétrequin et Pétrequin, 1988) d'une augmentation du degré de consommation des poignards avec l'accroissement des importations. Difficile en effet d'imaginer le stockage et la transmission sur plusieurs siècles de spécimens d'une remarquable fraîcheur (macro et microscopique), qui n'auraient pas gardé sur leurs surfaces les marques d'un tel traitement. Cette très probable longue durée d'utilisation implique également que nous sommes ici face à une sépulture très certainement collective, ce que suggérait d'ailleurs le nombre de lames de poignards. Cela signifie encore que la fosse sépulcrale devait être signalée dans le paysage, rôle qui a pu être tenu par le bloc de gneiss émergeant et accessible aux diverses manipulations sépulcrales. Elle implique enfin une occupation pérenne du plateau durant tout le troisième millénaire.

\section{VERS UNE INTERPRÉTATION TERRITORIALE}

Le Néolithique final (au sens très large du terme) est particulièrement bien représenté dans la région. Un récent programme de prospection thématique nous a permis de montrer que sur plus d'une centaine de sites ou indices de sites mésolithiques et néolithiques recensés dans les monts de Châlus, plus de soixante peuvent être attribués au Néolithique final (fig. 16; Perrin, 2000). L'intensité de l'occupation du territoire étudié explose littéralement pendant cette période. Contrairement à ce que l'on pressent pour les phases précédentes, où les sites sont principalement représentés sur les hauteurs, au troisième millénaire, les sites d'habitat descendent massivement dans les vallées. Sur l'ensemble des monts de Châlus, il est même possible de distinguer plusieurs zones de concentration (fig. 16 et 17), l'une centrée sur le bassin de la Tardoire, l'autre

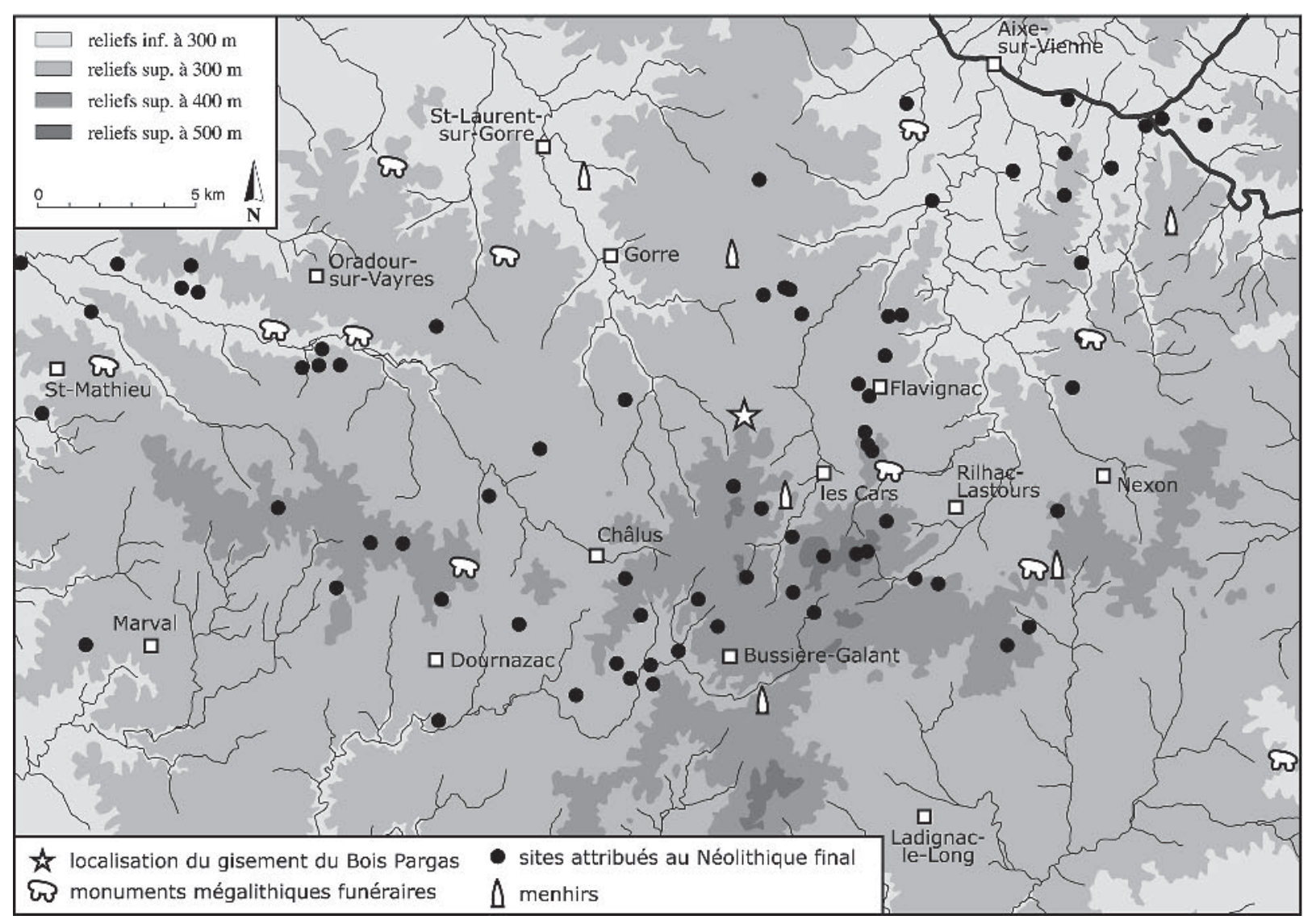

Fig. 16 - Carte générale des gisements du Néolithique final (au sens large) dans la région des monts de Châlus (Haute-Vienne), d'après les résultats des prospections systématiques et thématiques effectuées dans la région. 


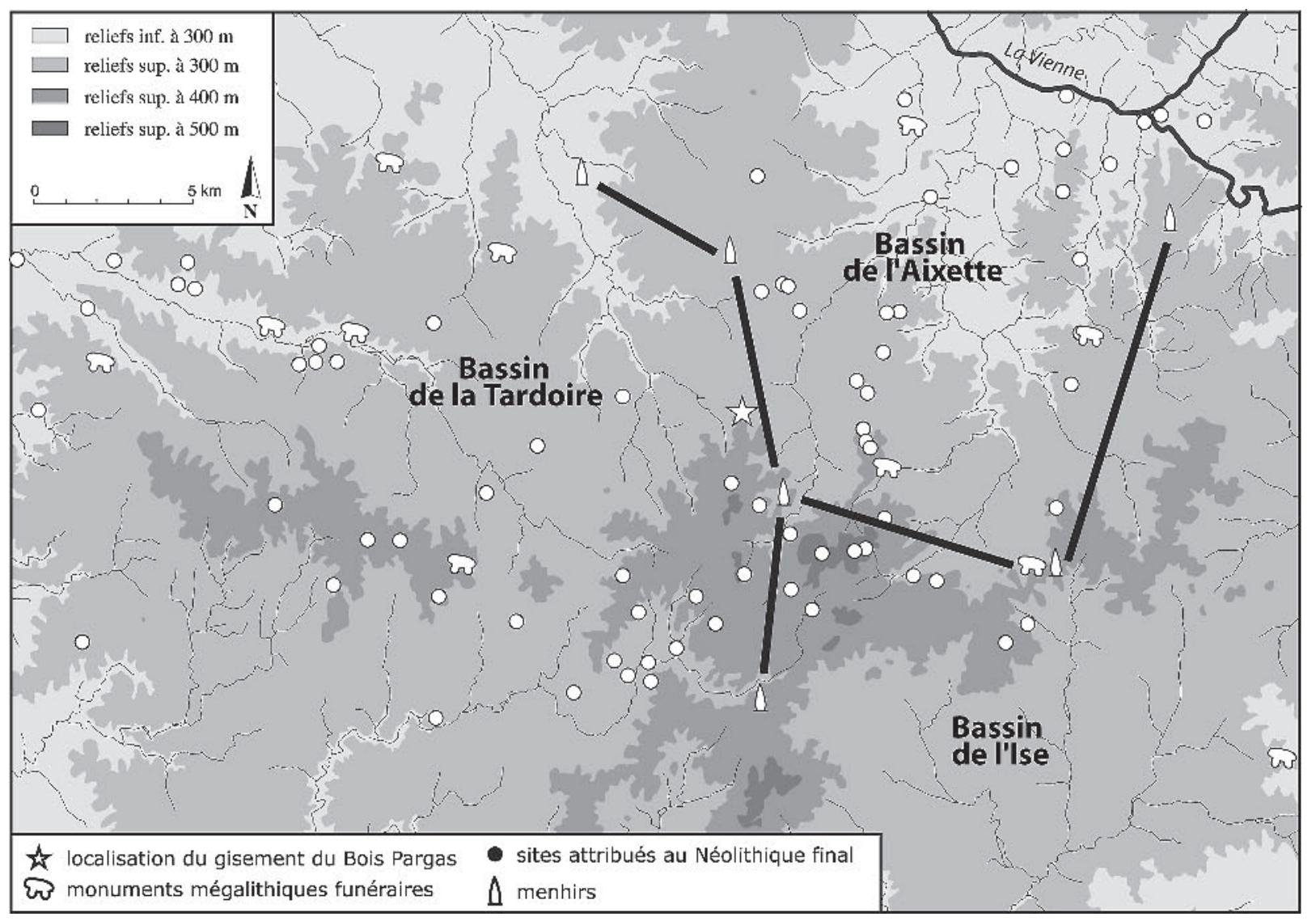

Fig. 17 - Hypothèse de structuration territoriale, montrant la possible individualisation de bassins versants délimités par des menhirs, regroupant sites d'habitats et monuments mégalithiques.

sur celui de l'Aixette, bassin qui s'ouvre ensuite sur celui de la Vienne. Entre ces deux zones de concentration principales viennent se placer quelques monuments mégalithiques, dolmens, tumulus et menhirs. Bien que l'exercice reste périlleux eu égard à tous les problèmes de conservation différentielle des gisements d'une part, et à l'imprécision générale des données chronologiques pour des sites documentés principalement par prospection d'autre part, on peut cependant suggérer une interprétation en termes territoriaux. En effet, on remarque que les monuments mégalithiques, notamment les menhirs, peuvent être compris comme délimitant plus ou moins fidèlement les bassins versants sus cités (fig. 17). Ainsi, sur les diverses pentes des monts de Châlus ont pu se développer des communautés néolithiques au sein de territoires centrés sur des petits bassins versants, plus ou moins bien délimités par des monuments mégalithiques de type menhir, et incluant en leurs seins des sites d'habitat de tailles diverses ainsi que des monuments funéraires. Ce «modèle» reste, bien entendu, très hypothétique et demande à être affiné, puisqu'en fonction de la ligne que l'on dessine entre le menhir de Bord (Les Cars) et le menhir de la Pierre Levée (Séreilhac), le site du Bois Pargas peut appartenir tant au territoire du bassin de l'Aixette qu'à celui de la Tardoire... Pour l'anecdote, remarquons d'ailleurs qu'une des parcelles voisines du Bois Pargas, légèrement plus occidentale, se dénomme aujourd'hui «Bois de la Pierre Pointue». Les ravages de la tempête de 1999 nous ont cependant empêchés pour l'instant d'aller vérifier sur place la présence ou non d'un éventuel menhir...

\section{CONCLUSION}

Le gisement archéologique du Bois Pargas s'avère ainsi être un site régionalement exceptionnel, notamment par le mobilier archéologique qu'il a pu fournir. Au terme de cette opération, nous avons pu montrer la présence, sur ce plateau, d'une fosse sépulcrale collective, «pseudo-mégalithique», du troisième millénaire avant notre ère. Si nous n'avons pas d'informations quant aux défunts eux-mêmes, le mobilier archéologique traduit très certainement un statut privilégié au sein de la société (Delcourt-Vlaeminck, 2002; Salanova, 1998). Il montre de plus l'insertion du gisement au sein de réseaux d'échanges à longue distance, traduisant par là une occupation marquée et durable du territoire à cette époque. Cela est d'ailleurs confirmé par les nombreux indices de sites déjà identifiés du Néolithique final dans les monts de Châlus (Conte et Perrin, 1998; Perrin, 2000). Cette abondance de documentation laisse espérer la possibilité de mise en place de recherches sur les territoires préhistoriques et les modalités d'occupation dans cette région au troisième millénaire. 


\section{NOTES}

(1) ArchéA, 26, route de Nexon, 87000 Limoges, archea@ archea.net (2) Ce sondage a été réalisé en juin 2001 grâce à la participation bénévole de Claire Chabroux, Jean-Pierre Château, Patrice Conte, Marie-Hélène Dias-Merinho, Christian Giraud, Jean-Claude Grany, Boris Hollemaert, Thierry Juste, Claire Manen, Julie Molard, Denis Ségalat et Thierry Sauret. Que tous soient ici remerciés de leur précieuse collaboration.

(3) Le gisement est localisé sur une parcelle propriété de monsieur A. Chazelas, de Saint-Martin-le-Vieux, à qui nous exprimons notre reconnaissance pour nous avoir autorisé, lui et son fils, à entreprendre ce sondage.

(4) La détermination des matières premières a fait l'objet d'un examen microscopique par Nicole Mallet, confirmant et affinant l'origine pressignienne de la majorité des pièces lithiques. Pierrick Fouéré a également procédé à un examen macroscopique de ces outils, suggérant notamment une possible origine aquitaine pour la lame non pressignienne. Que tous les deux soient remerciés de leurs aide et conseils.

\section{RÉFÉRENCES BIBLIOGRAPHIQUES}

AUBRY T. (1991) - L'exploitation des ressources en matières premières lithiques dans les gisements solutréens et badegouliens du Bassin versant de la Creuse (France), thèse de doctorat, université de Bordeaux I, Bordeaux, inédit, $327 \mathrm{p}$

BARGE H. (1982) - Les parures du Néolithique ancien au début de l'Âge des Métaux en Languedoc, éd. du CNRS, Paris, 399 p.

BEAUSOLEIL J.-M., PICARD P. (1987) - Un monument mégalithique à occupation artenacienne : le dolmen de «Chez Boucher» à La Croixsur-Gartempe, Haute-Vienne, Revue archéologique du Centre de la France, t. $26, \mathrm{n}^{\circ} 1$, p. $7-20$

BEUGNIER V. (1997) - L'usage du silex dans l'acquisition et le traitement des matières animales dans le Néolithique de Chalain et Clairvaux. La Motte-aux-Magnins et Chalain 3 (Jura, France). 3700-2980 av. J.-C., thèse de doctorat, université de Paris X-Nanterre, Paris, 464 p., inédit.

BEUGNIER V., PLISSON H. (2004) - Les poignards en silex du GrandPressigny: fonction de signe et fonction d'usage, in P. Bodu et C. Constantin dir., Approches fonctionnelles en Préhistoire, Actes du $X X V^{e}$ congrès préhistorique de France, Nanterre, 24-26 novembre 2000, Société préhistorique française, Paris, p. 139-154.

BURNEZ C., FOUÉRÉ P., LOUBOUTIN C. (1998) - Artenac et Campaniforme dans le Centre-Ouest de la France, Bulletin de la Société préhistorique française, t. $95, \mathrm{n}^{\circ} 3$, p. 303-304.

BURNEZ C., FOUÉRÉ P. dir. (1999) - Les enceintes néolithiques de Diconches à Saintes (Charente-Maritime). Une périodisation de l'Artenac, Société préhistorique française, Paris, Mémoire XXV, et Association des publications Chauvinoises, Mémoire XV, 2 vol., 829 p.

CHEVILLOT C. (1976) - Un atelier de bracelets en lignite décorés à Chalucet Saint-Jean-Ligoure, Haute-Vienne, Bulletin de la Société préhistorique française, t. 73, Études et Travaux, p. 422-436.

CHEVILLOT C. (1989) - Sites et cultures de l'Âge du Bronze en Périgord. Du groupe d'Artenac au groupe de Vénat, coll. Archéologies, éd. Vésuna, Périgueux, 2 vol., 248 p., 40 fig., 370 pl.

COLLIN F., MATTART D., PIRNAY L., SPECKENS J. (1991) - L'obtention du feu par percussion : approche expérimentale et tracéologique, Bulletin des Chercheurs de la Wallonie, t. XXXI, p. 19-49.

CONTE P., PERRIN T. (1998a) - Rapport de prospection diachronique. Communes de Rilhac-Lastours et Les Cars, Haute-Vienne, service régional de l'Archéologie Limousin, Limoges, inédit, 167 p., 48 fig.

CONTE P., PERRIN T. (1998b) - Recherches sur l'occupation humaine du territoire des communes des Cars et de Rilhac-Lastours, archéologie d'une forêt, Bulletin de la Société archéologique et historique du Limousin, t. CXXVI, p. 282-285.

CONTE P., GRANY J.-C., PERRIN T. (2001) - Premiers résultats des opérations archéologiques en forêt de Lastours-Les Cars, HauteVienne : peuplement, forêt et paysage du Mésolithique à l'époque moderne, Mémoire du Pays d'Aixe, t. 12, p. 1-34.

CORDIER G., RICQUET R., BRABAN H., POULAIN T. (1972) - Le site archéologique du dolmen de Vilaine à Sublaine (Indre-et-Loire), Gallia Préhistoire, t. 15, p. 31-135.

CORDIER G. (1986) - Les dépôts de lames de silex en France, Études préhistoriques, t. 17, p. 33-48.
DELAGE J.-P. (1993) - Les ateliers de taille du silex en Bergeracois durant le Néolithique, mémoire de DEA, École des hautes Études en sciences sociales, Toulouse, inédit, 103 p., 38 fig.

DELCOURT-VLAEMINCK M. (2002) - Le silex du Grand-Pressigny dans le Nord-Ouest de l'Europe. Le silex tertiaire, concurrent possible du Grand-Pressigny?, Presses universitaires du Septentrion, Lille, 601 p., 119 fig.

DELCOURT-VLAEMINCK M. (2004) - Les exportations du silex du Grand-Pressigny et du matériau tertiaire dans le Nord-Ouest de l'Europe au Néolithique final/Chalcolithique, in $\mathrm{M}$. Vander Linden et L. Salanova dir., Le troisième millénaire dans le Nord de la France et en Belgique, Mémoire XXXV, Société préhistorique française, Paris, p. $139-154$.

DELPORTE H. (1966) - Informations archéologiques de la circonscription Auvergne et Limousin, Gallia Préhistoire, t. 9, fasc. 2, p. 506-531.

FOUÉRÉ P. (1994) - Les industries en silex entre Néolithique moyen et Campaniforme dans le nord du Bassin aquitain. Approche méthodologique, implications culturelles de l'économie des matières premières et du débitage, thèse de doctorat, université de Bordeaux I, Bordeaux, inédit, 3 vol., 547 p.

GASSIN B. (1996) - Évolution socio-économique dans le Chasséen de la grotte de l'Église supérieure (Var). Apport de l'analyse fonctionnelle des industries lithiques, Monographie du CRA, $\mathrm{n}^{\circ} 17$, éd. CNRS, Paris, $326 \mathrm{p}$.

GESLIN M., BASTIEN G., MALLET N., FRESLIER B., GENTY P. (1982) - Le petit atelier de taille de la Creusette, commune de Barrou, Indre-et-Loire, Bulletin de la Société préhistorique française, t. 79, $\mathrm{n}^{\circ} 10-12$, p. 399-410.

HAMON T. (1999) - Architectures et culture de l'Artenac en Berry. Le camp des Chateliers à Moulins-sur-Céphons (Indre), service régional de l'Archéologie Centre, Orléans, inédit, 41 p.

HONEGGER M. (2001) - L'industrie lithique taillée du Néolithique moyen et final de Suisse, Monographies du CRA, $n^{\circ} 24$, éd. CNRS, Paris, 353 p., 198 fig.

HONEGGER M. (2006) - Grandes lames et poignards dans le Néolithique final du nord des Alpes, in J. Vaquer et F. Briois dir., La fin de l'Âge de Pierre en Europe du Sud. Matériaux et productions lithiques taillées remarquables dans le Néolithique et le Chalcolithique du Sud de l'Europe, Actes de la table ronde de l'EHESS, Carcassonne, 5-6 septembre 2003, Archives d'Écologie préhistorique, Toulouse, p. 43-56.

IHUEL E., avec la collaboration de MALLET N. et LOUBOUTIN C. (2002) - Les collections pressigniennes du musée des Antiquités nationales de Saint-Germain-en-Laye, Antiquités nationales, p. 29-76.

IHUEL E. (2004) - La diffusion du silex du Grand-Pressigny dans le Massif armoricain au Néolithique, coll. Documents préhistoriques $\mathrm{n}^{\circ} 18$, suppl. $\mathrm{n}^{\circ} 2$, CTHS et Bulletin de l'Association des Amis du Musée du Grand-Pressigny, Tours, 202 p.

IHUEL E., PELEGRIN J. (à paraître) - Du Jura au Poitou en passant par le Grand-Pressigny : une méthode de taille et des poignards particuliers vers 3000 av. J.-C., Les industries lithiques taillées des $I V^{t}$ et III ${ }^{e}$ millénaires en Europe occidentale, Actes de la table ronde de Toulouse, 7-9 avril 2005.

JOUSSAUME R. (1977) - Le mégalithe de la Pierre-Virante à XantonChassenon (Vendée), L'Anthropologie, vol. 81, p. 5-62. 
JOUSSAUME R. dir. (1998) - Les premiers paysans du golfe. Le Néolithique dans le marais Poitevin, éd. Patrimoines et Médias, Chauray, $140 \mathrm{p}$.

JOUSSAUME R., FOUÉRÉ P., CRÉDOT R. et coll. (2002) - Dolmens des Quatre Routes et de Bois Neuf III à Marsac, Creuse, Bulletin de la Société préhistorique française, t. $99, \mathrm{n}^{\circ} 1$, p. 49-80.

LAVIER C. (1996) - Dendrochronologie appliquée à l'archéologie : élaboration d'une chronologie du chêne (Quercus sp.) pour le Néolithique à partir des sites lacustres de Clairvaux-les-Lacs et de Chalain (Jura, France). Éléments pour l'estimation de la durée d'occupation des villages, mémoire de DEA, université de Franche-Comté, Besançon, 65 p., inédit.

LECORNEC J. (1996) - L'allée couverte de Bilgroix, Arzon, Morbihan, Bulletin de la Société polymathique du Morbihan, p. 15-64.

LOUBOUTIN C., BURNEZ C., CONSTANTIN C., SIDÉRA I. (1997) Beaumont-La Tricherie (Vienne) et Challignac (Charente) : deux sites d'habitat de la fin du Néolithique, Antiquités nationales, ${ }^{\circ} 29$, p. 4964.

MALLET N. (1992) - Le Grand-Pressigny : ses relations avec la civilisation Saône-Rhône, suppl. au Bulletin de la Société des amis du GrandPressigny, Argenton-sur-Creuse, 2 vol., 218 p., 100 fig., 123 pl.

MALLET N., PELEGRIN J., REDURON-BALLINGER M. (1994) - Sur deux dépôts de lames pressigniennes : Moigny et Boutigny (Essonne), Bulletin des Amis du Musée de Préhistoire du Grand-Pressigny, $\mathrm{n}^{\circ}$ 45, p. 25-37.

MARQUET J.-C., MILLET-RICHARD L.-A. (2000) - Les sites préhistoriques de la Touraine du sud, Hors-série $\mathrm{n}^{\circ} 2$ des Cahiers de la Claise Tourangelle, Archéa, 83 p.

MASSET C. (1997) - Les dolmens. Sociétés néolithiques et pratiques funéraires, Errance, Paris, 175 p.

MAUDUIT J., TARRÊTE J., TABORIN Y., GIRARD C. (1977) - La sépulture collective mégalithique de l'usine Vivez à Argenteuil, Vald'Oise, Gallia Préhistoire, t. 20, p. 177-227.

NOUEL A., DAUVOIS M., BAILLOUD G. (1965) - L'ossuaire néolithique d'Éteauville (commune de Lutz-en-Dunois, Eure-et-Loir), Bulletin de la Société préhistorique française, t. LXII, p. 576-648.

PATTE É. (1971) - Quelques sépultures du Poitou, du Mésolithique au Bronze moyen, Gallia Préhistoire, t. XIV, fasc. 1, p. 139-244.

PATTON M. (1995) - Neolithic Communities of the Channel Island, British Archaeological Review, ${ }^{\circ}$ 240, Oxford, 194 p.

PELEGRIN J. (à paraître) - Notes technologiques sur les pièces en silex du Grand-Pressigny de Chalain-Clairvaux rapportées à la fin du $4^{\mathrm{e}}$ millénaire et au tout début du $3^{\mathrm{e}}$ millénaire, in P. Pétrequin et A.-M. Pétrequin dir., Les sites littoraux néolithique de Clairvaux et Chalain (Jura). IV : du Ferrières au groupe de Clairvaux $\left(31^{e}\right.$ et $30^{e}$ siècle av. J.-C.), coll. Archéologie et culture matérielle, Maison des sciences de l'Homme, Paris.

PELEGRIN J., IHUEL E. (2005) - Les 306 nucléus de la ruine de la Claisière (Abilly, Indre-et-Loire), Bulletin des Amis du Musée du Grand-Pressigny, ${ }^{\circ}$ 56, p. 45-65.

PÉRIDY P. (1999) - Les enceintes néolithiques à fossés interrompus entre Loire et marais Poitevin, Bulletin de la Société préhistorique française, t. 96, p. 421-426.

PÉRIDY P. (2005) - Site d'enceintes néolithiques de la Chevêtelière, commune de L'Ile-d'Olonne et de Saint-Mathurin (Vendée), service régional de l'Archéologie des Pays de la Loire, Nantes, 32 p., inédit.

PERRIN T. (2000) - La Préhistoire récente dans les monts de Châlus. Chronologie et implantation des habitats néolithiques. Rapport de prospection thématique 1999-2000, service régional de l'Archéologie du Limousin, Limoges, inédit, 171 p.

PERRIN T. (2003) - Évolution du silex taillé dans le Néolithique hautrhodanien autour de la stratigraphie du Gardon (Ambérieu-en-Bugey Ain), Presses universitaire du Septentrion, Lille, 1016 p.
PÉTREQUIN P. dir. (1997) - Les sites littoraux néolithiques de Clairvaux-les-Lacs et de Chalain (Jura). III. Chalain station 3, 3200$2900 a v$. J.-C., Maison des sciences de 1'Homme, Paris, 2 vol., $765 \mathrm{p}$.

PÉTREQUIN A.-M., PÉTREQUIN P. (1988) - Le Néolithique des lacs. Préhistoire des lacs de Chalain et de Clairvaux (4000-2000 av. J.-C.), Errance, Paris, 284 p.

PÉTREQUIN A.-M., PÉTREQUIN P. (1990) - Flèches de chasse, flèches de guerre. Le cas des Danis d'Irian Jaya (Indonésie), Bulletin de la Société préhistorique française, t. 87, p. 484-511.

PLISSON H., BEUGNIER V. (2004) - Les poignards en silex du GrandPressigny : fonction de signe et fonctions d'usage, in P. Bodu, C. Constantin dir., Approches fonctionnelles en Préhistoire, $X X V^{e}$ congrès préhistorique de France, Nanterre, 24-26 novembre 2000, Société préhistorique française, Paris, p. 139-154.

SALANOVA L. (1998) - Le statut des assemblages campaniformes en contexte funéraire : la notion de «bien de prestige», Bulletin de la Société préhistorique française, t. 95, p. 315-326.

TIXIER J. (1977) - Informations archéologiques de la circonscription du Limousin, Gallia Préhistoire, t. 20, fasc. 2, p. 493-516.

VERGÉLY H. (2001) - Les objets de parure en jayet sur les Grands Causses aux périodes du Néolithique final et du Chalcolithique, mémoire de maîtrise, université de Toulouse-Le Mirail, Toulouse, 2 vol., 138 et 154 p., inédit.

VERGÉLY H. (2002) - Les parures en jayet des Grands Causses au Chalcolithique, Pirineus $i$ veïns al $3 r$ mil-lenni AC. De la fi del neolític a l'edat del bronze entre l'Ebre i la Garona. XII Col-loqui Internacional d'Arqueologia de Puigcerdà, 10-12 nov. 2000, Institut d'Estudis Ceretans, Puigcerdà i Andorra, p. 377-382.

VIELLET A. (2005) - Synthèse chronologique des bois d'œuvres des sites néolithiques de Clairvaux-les-Lacs et de Chalain (Jura, France). Les aléas de la méthode dendrochronologique, Bulletin de la Société préhistorique française, t. $102, \mathrm{n}^{\circ} 4$, p. 803-812.

VILLES A. (2003) - Que savons-nous des affinités culturelles du GrandPressigny au Néolithique récent et final?, Bulletin des Amis du Musée du Grand-Pressigny, ${ }^{\circ}$ 54, p. 43-74.

VILLES A. (2004) - Fouille de sauvetage sur l'atelier de taille pressignien «la Claisière» à Abilly (Indre-et-Loire), Internéo 5, Actes de la journée d'information du 20 novembre 2004, Société préhistorique française, Paris, p. 115-123.

VUAILLAT D. (1998) - Bref bilan documentaire sur le mégalithisme du Limousin, in P. Soulier dir., La France des dolmens et des sépultures collectives (4500-2000 avant J.-C.). Bilans documentaires régionaux, Archéologie Aujourd'hui, Errance, Paris, p. 179-185.

\section{Thomas PERRIN}

CNRS-UMR 5608-CRPPM

39, allées Jules-Guesde, 31000 Toulouse tperrin@free.fr

Ewen IHUEL

UMR 7055 «Préhistoire et Technologie» Maison de l'Archéologie et de l'Ethnologie 21, allée de l'Université, 92023 Nanterre Cedex ewen_ihuel@yahoo.fr

\section{Hugues PLISSON}

CNRS-UMR 6636

Maison méditerranéenne des sciences de l'Homme 5, rue du Château-de-l'Horloge BP 647, 13094 Aix-en-Provence Cedex 2 plisson@mmsh.univ-aix.fr 